\title{
Bifurcation currents in holomorphic dynamics on $\mathbf{P}^{k}$
}

\author{
Giovanni Bassanelli and François Berteloot*
}

\section{Introduction}

Potential theory has been introduced in one dimensional rational dynamics by Brolin and Tortrat (4 , 29]) but does not play a central role there. In higher dimension however, as the classical tools are not any longer efficient, pluri-potential theory has revealed itself to be essential. The fundamental works of Hubbard-Papadopol, Fornaess-Sibony, BedfordSmillie, Briend-Duval (see 26 for precise references) enlight the remarkable efficency of pluri-potential theory in holomorphic dynamics on $\mathbf{P}^{k}$ or $\mathbf{C}^{k}$. It is therefore tempting to study the parameter spaces in a similar way. More precisely, one would like to relate the bifurcations of an holomorphic family $\left\{f_{\lambda}\right\}_{\lambda \in X}$ of endomorphisms of $\mathbf{P}^{k}$ to the powers of a certain current on the parameter space $X$.

Let us recall that in dimension $k=1$, a bifurcation is said to occur at some point $\lambda_{0} \in X$ if the Julia set of $f_{\lambda}$ does not move continuously around $\lambda_{0}$. The famous work of Mane-SadSullivan [16], which is based on the $\lambda$-lemma and the Fatou-Cremer-Sullivan classification, relates the bifurcations with the instability of the critical orbits. It also establishes that the bifurcations concentrate on the complement of an open dense subset of $X$ (for the quadratic family $\left\{z^{2}+\lambda\right\}_{\lambda \in X=\mathbf{C}}$ the bifurcation locus is precisely the boundary of the Mandelbrot set).

A seminal idea towards the application of potential theory to the study of bifurcations is due to Przytycki who raised the following problem in the final remarks of his paper. Problem 22]: understand the connections between Lyapounov characteristics exponents and potential theory for rational mappings.

To support his point of view, Przytycki also analysed the following formula for a polynomial $p$ of degree $d$ on $\mathbf{C}$ (see also [17]):

$$
L(p)=\sum_{j} G_{p}\left(c_{j}\right)+\log d
$$

where $L(p)$ is the Lyapounov exponents of $p$ with respect to its equilibrium measure, $G_{p}$ its Green function and $c_{j}$ are the critical points of $p$. More recently, DeMarco ([8], 9]) has obtained a generalization of this formula to rational maps of $\mathbf{P}^{1}$. She also used her formula to show that, for an holomorphic family $\left\{f_{\lambda}\right\}_{\lambda \in X}$, the current $d d^{c} L\left(f_{\lambda}\right)$ is supported by

\footnotetext{
${ }^{*}$ The authors thank the Laboratoire E. Picard de Toulouse and the Dipartimento di Matematica di Parma for the reciprocal kind hospitality during the preparation of the paper. The first author was partially supported by GNSAGA of INDAM, the second one by the Université Italo-Française.
} 
the bifurcation locus.

The results of the present paper deal with Przytycki problem. In Section 2, we prove the

Theorem 2.2 Given an holomorphic family $\left\{f_{\lambda}\right\}_{\lambda \in X}$ of endomorphisms of $\mathbf{P}^{k}$, the function $L\left(f_{\lambda}\right)$, defined as the sum of Lyapounov exponents of $f_{\lambda}$ for its Green measure, is pluriharmonic on $X$ if the repulsive cycles of $f_{\lambda}$ move holomorphically on $X$.

This result indicates that $d d^{c} L\left(f_{\lambda}\right)$ is a reasonable bifurcation current in any dimension. Let us mention here that all what we need to know about $L$ in the paper is that $L(F)=$ $\int_{\mathbf{C}^{k+1}} \log \left|\operatorname{det} F^{\prime}\right| \mu_{F}$ where $F$ is a lift of $f$ and $\mu_{F}$ its Green measure.

With the goal of analysing the support of $d d^{c} L\left(f_{\lambda}\right)$ and its powers, we them generalize the formula (0.1) to endomorphisms of $\mathbf{P}^{k}$. This is done in a very natural way by using an integration by part formula on a suitable line bundle. We obtain the following

Formula (see Theorem 4.1). $L(f)=\sum_{j=0}^{k-1} \int_{C_{f}} g_{F}\left(d d^{c} g_{F}+\omega\right)^{j} \wedge \omega^{k-j-1}-$

$$
-\log d+\int_{\mathbf{P}^{k}} \log \left\|J_{F}\right\|_{0} \omega^{k}-(k+1)(d-1) \sum_{j=0}^{k} \int_{\mathbf{P}^{k}} g_{F}\left(d d^{c} g_{F}+\omega\right)^{j} \wedge \omega^{k-j} .
$$

For an holomorphic family $\left\{f_{\lambda}\right\}_{\lambda \in X}$, the above formula allows us to compute the bifurcation current $d d^{c} L\left(f_{\lambda}\right)$. We get the following synthetic statement

Theorem (see Corollary 4.6)

$$
d d^{c} L\left(f_{\lambda}\right)=p_{*}\left(\left(d d^{c} g_{F_{\lambda}}+\omega\right)^{k} \wedge\left[C_{X}\right]\right)
$$

and on $X \times \mathbf{P}^{k}$

$$
\left(d d^{c} g_{F_{\lambda}}+\omega\right)^{k+1}=0 .
$$

In these formulas $d d^{c}$ is acting on $X \times \mathbf{P}^{k}, p$ is the canonical projection from $X \times \mathbf{P}^{k}$ to $X, g_{F_{\lambda}}$ is the Green function of $f_{\lambda}$ on $\mathbf{P}^{k}$ associated to the lift $F_{\lambda}$ and $C_{X}:=\{(\lambda, z) \in$ $\left.X \times \mathbf{P}^{k} ; z \in C_{f_{\lambda}}\right\}$.

These results are established in Section 4 but, as the case of dimension $k=1$ is technically less involved and may help the reader to a better understanding, we treat it separetely in Section 3. It is worth emphasize that there is a certain interaction between the formulas (0.1) and (0.2) this may be seen in the example in Subsection 17.2 and in the Appendix (see formula (7.3)). Moreover, formula (0.3) is formally equivalent with the equation of geodesics on the space of Kahler metrics on $\mathbf{P}^{k}$, this leads to some examples of such geodesics as discussed in Subsection 7.1. Let us also stress that in the one dimensional case we have several explicit formulas for $L(f)$ (see Theorem 3.11). Moreover, our approach offers a much simpler proof of DeMarco's formula. The equivalence between DeMarco's formula and ours is a consequence of the following identity which may be of independant interest (see Proposition 4.9):

$$
\int_{\mathbf{P}^{1}} g_{F}\left(\mu_{f}+\omega\right)=\frac{1}{2}(\log |\operatorname{Res}(F)|-1) .
$$


Sections 5 and 6 are devoted to the study of bifurcations for holomorphic families $\left\{f_{\lambda}\right\}_{\lambda \in X}$ of rational maps of $\mathbf{P}^{1}$. For an holomorphic family $\left\{f_{\lambda}\right\}_{\lambda \in X}$ of endomorphisms of $\mathbf{P}^{1}$ we obtain a geometrical description of the support of the bifurcation currents $\left(d d^{c} L\left(f_{\lambda}\right)\right)^{p}$ by means of certain complex hypersurfaces.

For $\theta \in \mathbf{R} \backslash \mathbf{Z}$, the set of all $\lambda \in X$ such that $f_{\lambda}$ has a periodic point of period $n$ and multiplier $e^{2 i \pi \theta}$ is generically a complex hypersurface of $X$, denoted by $\operatorname{Per}\left(X, n, e^{2 i \pi \theta}\right)$. Therefore for $n$ fixed $\bigcup_{\theta} \operatorname{Per}\left(X, n, e^{2 i \pi \theta}\right)$ can be thought as a real hypersurface foliated by complex hypersurfaces. The union $\mathcal{Z}_{1}(X)$ of all these hypersurfaces is dense in the support of the the bifurcation current $d d^{c} L\left(f_{\lambda}\right)$ :

Theorem $\overline{\mathcal{Z}_{1}(X)}=\operatorname{Supp}\left(d d^{c} L\left(f_{\lambda}\right)\right)$.

We have included this geometrical characterization of the bifurcation locus in the statement of Theorem $\mathbf{5 . 2}$ which may be interpreted as treating a substantial part of ManeSad-Sullivan theory by pothentialist methods. The proof exploits the links between the vanishing of $d d^{c} L$, the motion of repulsive cycles and the stability of critical orbits. We point out that these links are revealed by formula (0.2) and the above quoted Theorem 2.2 .

For the powers of $d d^{c} L\left(f_{\lambda}\right)$ the geometry is more involved. Taking all the possible intersections between $p$ of the above complex hypersurface one gets a large family of codimension $p$ subvarieties of $X$; the union of this family, denoted by $\mathcal{Z}_{p}(X)$, satisfies:

Theorem [5.5] For any $1 \leq p \leq \operatorname{dim}_{\mathbf{C}} X$, Supp $\left(\left(d d^{c} L\left(f_{\lambda}\right)^{p}\right) \subset \overline{\mathcal{Z}_{p}(X)}\right.$.

These results have some significant consequences when considering the family $\mathcal{H}_{d}\left(\mathbf{P}^{1}\right)$ of all the rational maps of degree $d$. First of all one may show that

Proposition 6.5 For $1 \leq p \leq 2 d+1$ the bifurcation current $\left(d d^{c} L\right)^{p}$ has finite mass on $\mathcal{H}_{d}\left(\mathbf{P}^{1}\right)$.

This implies that $\left(d d^{c} L\right)^{2 d-2}$ induces a measure $\mu$ of finite mass on the moduli space $\mathcal{M}_{d}$ of rational maps of degree $d$. We call it the bifurcation measure and show that its support contains all isolated Lattès maps. Using our description of $\operatorname{Supp}\left(\left(d d^{c} L\right)^{p}\right)$ we also obtain the

Proposition 6.8 Any open set of $\mathcal{M}_{d}$ intersecting the support of the bifurcation measure contains an uncountable set of chaotic mappings.

As a by-product of our investigation one sees that any non flexible Lattès map is generating quite complicated bifurcations.

\section{Preliminaries}

In all the paper $\omega$ denotes the Fubini-Study form in $\mathbf{P}^{k}$ and \|\| be the Hermitian norm in $\mathbf{C}^{k+1}$.

\subsection{The spaces $\mathcal{H}_{d}\left(\mathbf{C}^{k+1}\right)$ and $\mathcal{H}_{d}\left(\mathbf{P}^{k}\right)$}

Every holomorphic endomorphism $f$ of $\mathbf{P}^{k}$ has a lift $F: \mathbf{C}^{k+1} \rightarrow \mathbf{C}^{k+1}$ that is: an homogeneous, non degenerate, polynomial map such that $\pi \circ F=f \circ \pi$, where 
$\mathbf{C}^{k+1} \backslash\{0\} \stackrel{\pi}{\rightarrow} \mathbf{P}^{k}$ is the canonical projection. The degree $d$ of $F$ is, by definition, the algebraic degree of $f$, while $d^{k}$ is the topological degree of $f$.

In the following it will always be assumed that $d \geq 2$.

Since an homogeneous polynomial of degree $d$ in $k+1$ variables depends on $\frac{(d+k) !}{d ! k !}$ coefficients, such a lift $F=\left(F_{0}, \ldots, F_{k}\right)$ can be identified with an element of $\mathbf{C}^{N+1}$, where $N=(k+1) \frac{(d+k) !}{d ! k !}-1$. With this identification, the space of all homogeneous, nondegenerate, polynomial maps of degree $d$ on $\mathbf{C}^{k+1}$ is an open subset of $\mathbf{C}^{N+1}$ which we shall denote by $\mathcal{H}_{d}\left(\mathbf{C}^{k+1}\right)$. Denoting again by $\mathbf{C}^{N+1} \backslash\{0\} \stackrel{\pi}{\rightarrow} \mathbf{P}^{N}$ the canonical projection, we get $\pi(F)=f$ and $\pi\left(\mathcal{H}_{d}\left(\mathbf{C}^{k+1}\right)\right)$ is the space of all holomorphic endomorphisms of $\mathbf{P}^{k}$ of degree $d$ which we shall denote by $\mathcal{H}_{d}\left(\mathbf{P}^{k}\right)$.

The following Proposition shows that the complement of $\mathcal{H}_{d}\left(\mathbf{C}^{k+1}\right)$ in $\mathbf{C}^{N+1}$ is a complex irreducible hypersurface $\tilde{\Sigma}_{d}=\{$ Res $=0\}$. Thus the projective hypersurface $\Sigma_{d}:=\pi\left(\tilde{\Sigma}_{d}\right)$ is the complement of $\mathcal{H}_{d}\left(\mathbf{P}^{k}\right)$ in $\mathbf{P}^{N}$.

Proposition 1.1 Let $F_{0}, \ldots, F_{k}$, be homogeneous polynomials of degree $d$, in $k+1$ complex variables. There exists a unique polynomial Res $\left(F_{0}, \ldots, F_{k}\right)$ in the coefficients of $F_{0}, \ldots, F_{k}$, which is homogeneous of degree $(k+1) d^{k}$, irreducible and such that

(i) $\operatorname{Res}\left(F_{0}, \ldots, F_{k}\right)=0$ if and only if $F=\left(F_{0}, \ldots, F_{k}\right): \mathbf{C}^{k+1} \rightarrow \mathbf{C}^{k+1}$ is degenerate,

(ii) $\operatorname{Res}\left(z_{0}^{d}, \ldots, z_{k}^{d}\right)=1$.

Proof. See [13] p. 427 and p. 105.

\subsection{Green functions}

To any $F \in \mathcal{H}_{d}\left(\mathbf{C}^{k+1}\right)$ is associated a Green function $G_{F}$ defined by

$$
G_{F}:=\lim _{n} d^{-n} \log \left\|F^{n}(z)\right\| \text {. }
$$

Let us stress that $G_{F}$ is the limit of a sequence $\left(G_{F, n}\right)_{n}$ of p.s.h and continuous functions on $\mathcal{H}_{d}\left(\mathbf{C}^{k+1}\right) \times\left(\mathbf{C}^{k+1} \backslash\{0\}\right)$. The following Proposition summarizes the regularity properties of $G_{F}(z)$. The only novelty here is the Hölder-continuity in $F$.

Proposition 1.2 i) For any compact subset $\mathcal{K}$ of $\mathcal{H}_{d}\left(\mathbf{C}^{k+1}\right)$, the sequence $G_{F, n}(z)$ converges uniformly to $G_{F}(z)$ on $\mathcal{K} \times\left(\mathbf{C}^{k+1} \backslash\{0\}\right)$. In particular $G_{F}(z)$ is p.s.h and continuous on $\mathcal{H}_{d}\left(\mathbf{C}^{k+1}\right) \times\left(\mathbf{C}^{k+1} \backslash\{0\}\right)$. It satisfies the following homogeneity property:

$$
G_{F}(t z)=\log |t|+G_{F}(z) ; \quad \forall t \in \mathbf{C}^{*}, \forall z \in \mathbf{C}^{k+1}
$$

and functional equation

$$
G_{F} \circ F=d G_{F} .
$$

In the definition of $G_{F}$, the norm \|\| may be replaced by any continuous jauge function.

ii) The function $G_{F}(z)$ is Hölder-continuous on every compact subset of $\mathcal{H}_{d}\left(\mathbf{C}^{k+1}\right) \times$ $\left(\mathbf{C}^{k+1} \backslash\{0\}\right)$. 


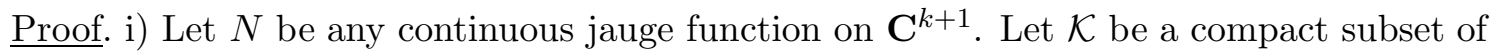
$\mathcal{H}_{d}\left(\mathbf{C}^{k+1}\right)$ and $C>1$ be a constant such that

$$
\frac{1}{C} \leq N(F(z)) \leq C ; \forall F \in \mathcal{K}, \forall z \in\{N=1\}
$$

Then, by homogeneity we have:

$$
\frac{1}{C^{1+\ldots+d^{n-1}}} N(z)^{d^{n}} \leq N\left(F^{n}(z)\right) \leq C^{1+\ldots+d^{n-1}} N(z)^{d^{n}} ; \forall F \in \mathcal{K}, \forall z \in \mathbf{C}^{k+1} \backslash\{0\}, \forall n \in \mathbf{N}
$$

After replacing $z$ by $F^{m}(z)$, taking log and dividing by $d^{n+m}$, (1.2) gives:

$$
\left|G_{F, n+m}(z)-G_{F, n}(z)\right| \leq \frac{\log C}{d^{m}(d-1)} ; \forall F \in \mathcal{K}, \forall z \in \mathbf{C}^{k+1} \backslash\{0\} .
$$

ii) The Hölder continuity in $z$ has been established by Briend-Duval (3]). Inspecting the proof and taking into account the continuity of $G_{F}(z)$ in $(F, z)$, it is not hard to see that the constants might be chosen uniformly in $F$ (see 26] Théorème 1.7.1 and Remarque 1.7.2). More precisely, for any compact $\mathcal{K} \times K \subset \mathcal{H}_{d}\left(\mathbf{C}^{k+1}\right) \times\left(\mathbf{C}^{k+1} \backslash\{0\}\right)$ there are constants $C>0$ and $0<\alpha<1$ such that:

$$
\left|G_{F}(z)-G_{F}\left(z^{\prime}\right)\right| \leq C\left\|z-z^{\prime}\right\|^{\alpha} ; \forall F \in \mathcal{K}, \forall z, z^{\prime} \in K .
$$

We will show how this property may be transfered on $F$. We may assume that $\left\{G_{F}=\right.$ $0\} \subset K$ for every $F \in \mathcal{K}$. Let us pick $F, F_{0} \in \mathcal{K}$ and consider the jauge function $N_{0}:=e^{G_{F_{0}}}$. By the Hölder-continuity of $G_{F_{0}}$ we have

$$
\left|G_{F_{0}}(F(z))-G_{F_{0}}\left(F_{0}(z)\right)\right| \leq C_{1}\left\|F(z)-F_{0}(z)\right\|^{\alpha} \leq C_{2}\left\|F-F_{0}\right\|^{\alpha} ; \forall z \in K .
$$

When $z \in\left\{N_{0}=1\right\}$ this inequality becomes $\frac{1}{C_{0}} \leq N_{0}(F(z)) \leq C_{0}$ where $C_{0}:=$ $e^{C_{2}\left\|F-F_{0}\right\|^{\alpha}}$. Just like for (1.2) this implies

$\frac{1}{C_{0}^{1+\ldots+d^{n-1}}} N_{0}(z)^{d^{n}} \leq N_{0}\left(F^{n}(z)\right) \leq C_{0}^{1+\ldots+d^{n-1}} N_{0}(z)^{d^{n}} ; \forall F \in \mathcal{K}, \forall z \in \mathbf{C}^{k+1} \backslash\{0\}, \forall n \in \mathbf{N}$.

Taking log, dividing by $d^{n}$ and making $n \rightarrow \infty$, this yields (as $G_{F}=\lim _{n} d^{-n} N_{0}\left(F^{n}(z)\right)$ ):

$$
\left|G_{F}(z)-G_{F_{0}}(z)\right| \leq \frac{C_{2}}{d-1}\left\|F-F_{0}\right\|^{\alpha} ; \forall z \in\left(\mathbf{C}^{k+1} \backslash\{0\}\right) .
$$

The Green function $G_{F}$ induces a continous, $\omega$-p.s. $h$ function $g_{F}$ on $\mathcal{H}_{d}\left(\mathbf{P}^{k}\right) \times \mathbf{P}^{k}$ which will also be called a Green function of $F$ :

$$
g_{F} \circ \pi:=G_{F}-\log \|\| .
$$

Remark 1.3 It is straightforward to check that $g_{F}(z) \leq \frac{M}{d-1}$, where $M:=\sup _{\|z\|=1}\|F(z)\|$. In particular for every compact subset $\mathcal{K} \subset \mathbf{C}^{N}=\mathcal{H}_{d}\left(\mathbf{C}^{k+1}\right) \cup \Sigma_{d}, g_{F}$ is bounded from above on $\left(\mathcal{K} \cap \mathcal{H}_{d}\left(\mathbf{C}^{k+1}\right)\right) \times \mathbf{P}^{k}$. 


\subsection{Green currents and measures}

Let $f \in \mathcal{H}_{d}\left(\mathbf{P}^{k}\right)$ and $F \in \mathcal{H}_{d}\left(\mathbf{C}^{k+1}\right)$ a lift of $f$. One defines a closed, positive $(1,1)$-current $T_{f}$ on $\mathbf{P}^{k}$ by setting:

$$
T_{f}:=d d^{c} g_{F}+\omega
$$

As (1.3) shows, this current may equivalently be defined by $\pi^{*} T_{f}=d d^{c} G_{F}$. Since $g_{a F}=\frac{1}{d-1} \log |a|+g_{F}$, this current does not depend on the choice of the lift $F$ and will be called the Green current of $f$. The functional equation (1.1) implies that:

$$
f^{*} T_{f}=d T_{f}
$$

The Green measure $\mu_{f}$ of $f$ is defined by

$$
\mu_{f}:=\left(T_{f}\right)^{k}
$$

It is a probability measure with respect to which $f$ has constant Jacobian: $f^{*} \mu_{f}=d^{k} \mu_{f}$. It follows that $\mu_{f}$ is $f$-invariant $\left(f_{*} \mu_{f}=\mu_{f}\right)$ and $f$-ergodic.

It will also be usefull to consider the probability measures $m$ and $\mu_{F}$ defined on $\mathbf{C}^{k+1}$ by:

$$
m:=\left(d d^{c} \log ^{+}\|\|\right)^{k+1} \quad \mu_{F}:=\left(d d^{c} G_{F}^{+}\right)^{k+1}
$$

these measures are respectively supported by $\{\|\|=1\}$ and $\left\{G_{F}=0\right\}$; they are related to $\omega^{k}$ and $\mu_{f}$ by:

$$
\pi_{*} m=\omega^{k} \quad \pi_{*} \mu_{F}=\mu_{f} .
$$

\subsection{Lyapounov exponents}

Let $f \in \mathcal{H}_{d}\left(\mathbf{P}^{k}\right)$ and $F \in \mathcal{H}_{d}\left(\mathbf{C}^{k+1}\right)$ a lift of $f$. We shall denote by $L(F)$ the sum of Lyapounov exponents of $F$ with respect to $\mu_{F}$ and by $L(f)$ the sum of Lyapounov exponents of $f$ with respect to $\mu_{f}$. The following facts hold:

(i) $L(F)=\int_{\mathbf{C}^{k+1}} \log \left|\operatorname{det} F^{\prime}\right| \mu_{F}($ see [3]);

(ii) $L(F)=L(f)+\log d($ see [14]);

(iii) $L\left(F^{n}\right)=n L(F)$, for all $n \in \mathbf{N}^{*}$ (use (i) and $f_{*} \mu_{f}=\mu_{f}$ );

(iv) $L(f)$ is p.s.h. on $\mathcal{H}_{d}\left(\mathbf{C}^{k+1}\right)$, as it has been proved in the larger setting of polynomial like mappings (see [10]).

\subsection{Green metric on $\mathcal{O}_{\mathbf{P}^{k}}(D)$}

Let $D \in \mathbf{N}^{*}$. The line bundle $\mathcal{O}_{\mathbf{P}^{k}}(D)$ over $\mathbf{P}^{k}$ is conveniently seen as the quotient of $\left(\mathbf{C}^{k+1} \backslash\{0\}\right) \times \mathbf{C}$ by the relation $(z, x) \equiv\left(u z, u^{D} x\right)$ for all $u \in \mathbf{C}^{*}$, its elements beeing denoted $[z, x]$. The canonical metric on $\mathcal{O}_{\mathbf{P}^{k}}(D)$ may be expressed by:

$$
\|[z, x]\|_{0}:=e^{-D \log \|z\|}|x| .
$$


The homogeneity of $G_{F}$ allows us to associate to any $F \in \mathcal{H}_{d}\left(\mathbf{C}^{k+1}\right)$ a Green metric defined on $\mathcal{O}_{\mathbf{P}^{k}}(D)$ by:

$$
\|[z, x]\|_{G_{F}}:=e^{-D G_{F}}|x| .
$$

The main interest of endowing $\mathcal{O}_{\mathbf{P}^{k}}(D)$ with such a metric is to produce a very usefull formula for $L(F)$. To this purpose we will denote $J_{F}$ the holomorphic section induced by $\operatorname{det} F^{\prime}$ on $\mathcal{O}_{\mathbf{P}^{k}}(D)$ for $D=(k+1)(d-1)$ :

$$
J_{F} \circ \pi=\left[z, \operatorname{det} F^{\prime}(z)\right] ; \quad \forall z \in \mathbf{C}^{k+1} .
$$

Then we have the following lemma:

Lemma 1.4 Let $F \in \mathcal{H}_{d}\left(\mathbf{P}^{k}\right)$ and $D=(k+1)(d-1)$. Let us endow $\mathcal{O}_{\mathbf{P}^{k}}(D)$ with the canonical and the Green metrics. Then the following identities occur:

1) $L(F)=\int_{\mathbf{C}^{k+1}} \log \left|\operatorname{det} F^{\prime}\right| \mu_{F}=\int_{\mathbf{P}^{k}} \log \left\|J_{F}\right\|_{G_{F}} \mu_{f}$

2) $\int_{\mathbf{C}^{k+1}} \log \left|\operatorname{det} F^{\prime}\right| m=\int_{\mathbf{P}^{k}} \log \left\|J_{F}\right\|_{0} \omega^{k}$.

Proof. 1) As $G_{F}$ identically vanishes on the support of $\mu_{F}$, we have $\int_{\mathbf{C}^{k+1}} \log \left|\operatorname{det} F^{\prime}\right| \mu_{F}=$ $\int_{\mathbf{C}^{k+1}} \log \left(e^{-D G_{F}}\left|\operatorname{det} F^{\prime}\right|\right) \quad \mu_{F}=\int_{\mathbf{C}^{k+1}} \log \left\|J_{F} \circ \pi\right\| \mu_{F}$ and the conclusion follows from $\pi_{*} \mu_{F}=\mu_{f}$.

2) We proceed analogously, using the fact that $\log ^{+}\|\|$identically vanishes on the support of $m$ and $\pi_{*} m=\omega^{k}$.

\section{A current detecting the holomorphic motion of repulsive cycles}

Let $\left\{f_{\lambda}\right\}_{\lambda \in X}$ be an holomorphic family of endomorphisms of $\mathbf{P}^{k}$ parametrized by a complex manifold $X$. The p.s.h. function $L(\lambda)=L\left(f_{\lambda}\right)$ given by the sum of Lyapounov exponents of $f_{\lambda}$, furnishes a closed, positive $(1,1)$-current $d d^{c} L$ on $X$. In this section, we will show that $d d^{c} L$ vanishes where the repulsive cycles of $f_{\lambda}$ move holomorphically. Let us precisely state what we mean by this holomorphic motion.

Definition 2.1 The repulsive cycles of period $n$ of $\left\{f_{\lambda}\right\}_{\lambda \in X}$ move holomorphically over an open subset $U$ of $X$ if and only if there exists a collection of holomorphic mappings $\alpha_{n, j}: U \rightarrow \mathbf{P}^{k}$ such that, for any $\lambda \in U$, the set of $n$-repulsive cycles is given by $\left\{\alpha_{n, j}(\lambda)\right\}$.

In dimension $k=1$, it is well known that the Julia set of $f_{\lambda}$ depends continuously on $\lambda \in U$ if and only if the repulsive cycles of sufficently high order of $f_{\lambda}$ move holomorphically on $U$ (see 18, Theorem 4.2).

Although such a phenomenon is far from beeing clear in higher dimension, we would like to motivate the study of $d d^{c} L$ as a bifurcation current by the following result:

Theorem 2.2 Let $\left(f_{\lambda}\right)_{\lambda \in X}$ be an holomorphic familly of endomorphisms of $\mathbf{P}^{k}$ with algebraic degree $d$. If all repulsive cycles of $f_{\lambda}$ of period $n \geq n_{0}$ move holomorphically on some open subset $U$ of $X$ then the sum $L\left(f_{\lambda}\right)$ of Lyapounov exponents of $f_{\lambda}$ is pluriharmonic on $U$. 
Proof. We may assume that $U$ is a small ball on which $\left\{f_{\lambda}\right\}_{\lambda \in U}$ lifts to some holomorphic family $\left\{F_{\lambda}\right\}_{\lambda \in U}$. Then it is not hard to see that the set of $n$-repulsive cycles of $F_{\lambda}$ is given by $\left\{a_{n, j}(\lambda) ; \lambda \in U\right\}$ where the maps $a_{n, j}: U \rightarrow \mathbf{C}^{k+1}$ are holomorphic. The number of elements of $\left\{a_{n, j}(\lambda) ; \lambda \in U\right\}$ does not depend on $\lambda$ and will be denoted $N_{n}$.

By a theorem of Briend-Duval (see [3], Theorem 2), the Green measure $\mu_{F_{\lambda}}$ of $F_{\lambda}$ is the weak limit of a sequence of discrete measures:

$$
\frac{1}{N_{n}} \Sigma_{j} \delta_{a_{n, j}(\lambda)}=: \mu_{F_{\lambda}, n} \rightarrow \mu_{F_{\lambda}} .
$$

It is therefore natural to consider the sequence of pluriharmonic functions

$$
L_{n}(\lambda):=\int_{\mathbf{C}^{k+1}} \log \left|\operatorname{det} F_{\lambda}^{\prime}\right| \mu_{F_{\lambda}, n}=\frac{1}{N_{n}} \Sigma_{j} \log \left|\operatorname{det} F_{\lambda}^{\prime}\left(a_{n, j}(\lambda)\right)\right|
$$

and to compare it with $L(\lambda):=\int_{\mathbf{C}^{k+1}} \log \left|\operatorname{det} F_{\lambda}^{\prime}\right| \mu_{F_{\lambda}}=L\left(f_{\lambda}\right)+\log d$.

However, as the functions $\log \left|\operatorname{det} F_{\lambda}^{\prime}\right|$ is unbounded, this comparison is not immediate. As we shall see, the fact that the measures $\pi_{*} \mu_{F_{\lambda}}$ have local $\alpha$-Hölder potentials is essential to overcome this difficulty.

Let us now enter into details and, to this purpose, fix a few notations. The Green function of $F_{\lambda}$ will be denoted $G_{\lambda}$ and for any $\varepsilon>0$ we set

$$
W_{\lambda, \varepsilon}:=\left\{G_{\lambda}=0\right\} \cap\left\{\left|\operatorname{det} F_{\lambda}^{\prime}\right| \leq \varepsilon\right\} .
$$

We shall write $d_{n, j}(\lambda)$ the holomorphic function $\operatorname{det} F_{\lambda}^{\prime}\left(a_{n, j}(\lambda)\right)$ and introduce the following sequence of discrete measures:

$$
\mathcal{L}_{n, \lambda}:=\frac{1}{N_{n}} \sum_{j} \log \left|d_{n, j}(\lambda)\right| \delta_{a_{n, j}(\lambda)}
$$

Since $G_{\lambda} \circ F_{\lambda}^{n}=d^{n} G_{\lambda}$, the $F_{\lambda}^{n}$-fixed points $a_{n, j}(\lambda)$ belong to $\left\{G_{\lambda}=0\right\}$ and thus, according to our notations, we have

$$
L_{n}(\lambda)=\mathcal{L}_{n, \lambda}\left(\mathbf{C}^{k+1}\right)=\mathcal{L}_{n, \lambda}\left(W_{\lambda, \varepsilon}\right)+\mathcal{L}_{n, \lambda}\left(W_{\lambda, \varepsilon}^{c}\right)
$$

We now fix $\lambda_{0} \in U, z_{0} \in \mathbf{C}^{N} \backslash\{0\}$ and $\rho>0$ such that $u_{\theta}:=\lambda_{0}+\rho e^{i \theta} z_{0}$ belongs to $\mathrm{U}$ for every $\theta \in \mathbf{R}$. Since the functions $L_{n}(\lambda)$ are pluriharmonic on $U$, the identity (2.1) may be rewritten as

$$
L_{n}\left(\lambda_{0}\right)=\frac{1}{2 \pi} \int_{0}^{2 \pi} \mathcal{L}_{n, u_{\theta}}\left(W_{u_{\theta}, \varepsilon}^{c}\right) d \theta+\frac{1}{2 \pi} \int_{0}^{2 \pi} \mathcal{L}_{n, u_{\theta}}\left(W_{u_{\theta}, \varepsilon}\right) d \theta
$$

The function $L$ being p.s.h. (see Subsection 1.4) we simply have to deduce from (2.2) that $\frac{1}{2 \pi} \int_{0}^{2 \pi} L\left(u_{\theta}\right) d \theta \leq L\left(\lambda_{0}\right)$. This will require the following lemmas.

Lemma 2.3 There exists an universal function $\left.\left.\left.M:] 0, \varepsilon_{0}\right] \rightarrow\right] 0,1\right]$ which tends to 0 at 0 and such that $\frac{1}{2 \pi} \int_{0}^{2 \pi} \mathcal{L}_{n, u_{\theta}}\left(W_{u_{\theta}, \varepsilon}\right) d \theta \geq \mathcal{L}_{n, \lambda_{0}}\left(W_{\lambda_{0}, M(\varepsilon)}\right)$ for every $n \in \mathbf{N}$ and every $0<\varepsilon \leq \varepsilon_{0}$. 
Lemma $2.4 \lim _{\varepsilon \rightarrow 0}\left(\liminf _{n} \mathcal{L}_{n, \lambda}\left(W_{\lambda, \varepsilon}^{c}\right)\right)=L(\lambda)$ for every $\lambda \in X$.

Using Lemma 2.3 and the identities (2.1), (2.2) we get

$$
\begin{array}{r}
\frac{1}{2 \pi} \int_{0}^{2 \pi} \mathcal{L}_{n, u_{\theta}}\left(W_{u_{\theta}, \varepsilon}^{c}\right) d \theta=L_{n}\left(\lambda_{0}\right)-\frac{1}{2 \pi} \int_{0}^{2 \pi} \mathcal{L}_{n, u_{\theta}}\left(W_{u_{\theta}, \varepsilon}\right) d \theta \\
\leq L_{n}\left(\lambda_{0}\right)-\mathcal{L}_{n, \lambda_{0}}\left(W_{\lambda_{0}, M(\varepsilon)}\right)=\mathcal{L}_{n, \lambda_{0}}\left(W_{\lambda_{0}, M(\varepsilon)}^{c}\right)
\end{array}
$$

then, by Fatou's theorem we have

$$
\frac{1}{2 \pi} \int_{0}^{2 \pi} \liminf _{n} \mathcal{L}_{n, u_{\theta}}\left(W_{u_{\theta}, \varepsilon}^{c}\right) d \theta \leq \liminf _{n} \mathcal{L}_{n, \lambda_{0}}\left(W_{\lambda_{0}, M(\varepsilon)}^{c}\right)
$$

Thus, as $\lim _{\varepsilon \rightarrow 0} M(\varepsilon)=0$, the inequality $\frac{1}{2 \pi} \int_{0}^{2 \pi} L\left(u_{\theta}\right) d \theta \leq L\left(\lambda_{0}\right)$ immediately follows from Lemma 2.4 when $\varepsilon \rightarrow 0$. This ends the proof of Theorem 2.2 .

Proof of lemma 2.3, we shall use the following fact which is a direct consequence of Montel's theorem and Hurwitz lemma.

Fact: Let $0<\rho<r<R$ et $\mathcal{S}_{\varepsilon}:=\left\{\varphi \in \mathcal{O}\left(\Delta_{r}, \Delta_{R}^{*}\right) / \operatorname{In} f_{|z|=\rho}|\varphi(z)|=\varepsilon\right\}$. Let $M(\varepsilon):=$ $\operatorname{Sup}_{\varphi \in \mathcal{S}_{\varepsilon}} \operatorname{Sup}_{|z| \leq \rho}|\varphi(z)|$. Then $\lim _{\varepsilon \rightarrow 0} M(\varepsilon)=0$ and in particular $M(\varepsilon) \leq 1$ for $0<\varepsilon \leq \varepsilon_{0}$.

Let us observe that the functions $d_{n, j}(\lambda)$ are uniformly locally bounded. This follows from the continuity of $G_{\lambda}(z)$ and the previous observation that $\left\{a_{n, j}(\lambda)\right\} \subset\left\{G_{\lambda}=0\right\}$. According to our notations we have

$$
\frac{1}{2 \pi} \int_{0}^{2 \pi} d \theta \int_{W_{u_{\theta}, \varepsilon}} \mathcal{L}_{n, u_{\theta}}=\frac{1}{N_{n}} \sum_{j}^{\prime} \frac{1}{2 \pi} \int_{0}^{2 \pi} \log \left|d_{n, j}\left(u_{\theta}\right)\right| \mathbf{1}_{\left\{\left|d_{n, j}\right| \leq \varepsilon\right\}}\left(u_{\theta}\right) d \theta
$$

where $\Sigma_{j}^{\prime}$ indicates that we only consider the terms for which $\operatorname{In} f_{\theta}\left|d_{n, j}\left(u_{\theta}\right)\right| \leq \varepsilon$. By the Fact, all these terms satisfy $\left|d_{n, j}\left(u_{\theta}\right)\right| \leq M(\varepsilon) \leq 1$ for $\varepsilon \leq \varepsilon_{0}$ and $\left|u_{\theta}-\lambda_{0}\right| \leq \rho$. In particular, $\frac{1}{2 \pi} \int_{0}^{2 \pi} \log \left|d_{n, j}\left(u_{\theta}\right)\right| \mathbf{1}_{\left\{\left|d_{n, j}\right| \leq \varepsilon\right\}}\left(u_{\theta}\right) d \theta \geq \frac{1}{2 \pi} \int_{0}^{2 \pi} \log \left|d_{n, j}\left(u_{\theta}\right)\right| d \theta=\log \left|d_{n, j}\left(\lambda_{0}\right)\right|$. Thus (2.3) yields

$$
\frac{1}{2 \pi} \int_{0}^{2 \pi} \mathcal{L}_{n, u_{\theta}}\left(W_{u_{\theta}, \varepsilon}\right) d \theta \geq \frac{1}{N_{n}} \sum_{j}^{\prime} \log \left|d_{n, j}\left(\lambda_{0}\right)\right| .
$$

Finally, as $\left|d_{n, j}\left(\lambda_{0}\right)\right| \leq M(\varepsilon) \leq 1$ for all terms in $\Sigma_{j}^{\prime}$, we have

$$
\frac{1}{N_{n}} \sum_{j}^{\prime} \log \left|d_{n, j}\left(\lambda_{0}\right)\right| \geq \frac{1}{N_{n}} \sum_{j}^{\prime} \log \left|d_{n, j}\left(\lambda_{0}\right)\right| \mathbf{1}_{\left\{\left|d_{n, j}\right| \leq M(\varepsilon)\right\}}\left(\lambda_{0}\right)=\mathcal{L}_{n, \lambda_{0}}\left(W_{\lambda_{0}, M(\varepsilon)}\right)
$$

and the conclusion follows. 
Proof of lemma 2.4 Let us denote $\log _{\varepsilon}(x)$ a smooth function on $\left[0,+\infty\left[\right.\right.$ such that $\log _{\varepsilon}(x) \geq$ $2 \log \varepsilon$ for $0 \leq x<\varepsilon<1$ and $\log _{\varepsilon}(x)=\log (x)$ for $x \geq \varepsilon$. Then

$$
0 \leq \mathcal{L}_{n, \lambda}\left(W_{\lambda, \varepsilon}^{c}\right)-\int_{\mathbf{C}^{k+1}} \log _{\varepsilon}\left|\operatorname{det} F_{\lambda}^{\prime}\right| \mu_{F_{\lambda}, n} \leq-2(\log \varepsilon) \mu_{F_{\lambda}, n}\left(\left\{\left|\operatorname{det} F_{\lambda}^{\prime}\right| \leq \varepsilon\right\}\right) .
$$

There are constants $a, A>0$ such that $\left\{\left|\operatorname{det} F_{\lambda}^{\prime}\right| \leq \varepsilon\right\} \subset \pi^{-1}\left(V_{A \varepsilon^{a}}\left(C_{f_{\lambda}}\right)\right)$ where $V_{t}\left(C_{f_{\lambda}}\right)$ denotes a $t$-neighbourhood of $C_{f_{\lambda}}$. Since $\mu_{\lambda}:=\pi_{\star} \mu_{F_{\lambda}}$ has (local) $\alpha$-Hölder continuous potentials we have $\mu_{\lambda}\left(V_{A \varepsilon^{a}}\left(C_{f_{\lambda}}\right)\right) \leq c s t \varepsilon^{a \alpha}$ (see the proof of Theorem 1.7.3 in [26]). Thus, for $n$ big enough,

$$
\mu_{F_{\lambda}, n}\left(\left\{\left|\operatorname{det} F_{\lambda}^{\prime}\right| \leq \varepsilon\right\}\right) \leq 2 \mu_{F_{\lambda}}\left(\left\{\left|\operatorname{det} F_{\lambda}^{\prime}\right| \leq 2 \varepsilon\right\}\right) \leq \operatorname{cst} \varepsilon^{a \alpha} .
$$

From (2.4) and (2.5) we get

$$
0 \leq \liminf _{n} \mathcal{L}_{n, \lambda}\left(W_{\lambda, \varepsilon}^{c}\right)-\int_{\mathbf{C}^{k+1}} \log _{\varepsilon}\left|\operatorname{det} F_{\lambda}^{\prime}\right| \mu_{F_{\lambda}} \leq-c s t \varepsilon^{a \alpha} \log \varepsilon
$$

and the conclusion follows by making $\varepsilon \rightarrow 0$.

\section{Formulas for the Lyapounov exponent of a rational func- tion}

In this section we establish some formulas which relate the Lyapuonov exponent to the critical points of a rational function.

Theorem 3.1 Let $f$ be a rational function of degree $d$ and $F$ be one of its lifts to $\mathbf{C}^{2}$. The Lyapounov exponent $L(f)$ of $f$ is given by one of the following formulas:

(i) $L(f)+\log d=\int_{\mathbf{P}^{1}} g_{F}\left[C_{f}\right]-2(d-1) \int_{\mathbf{P}^{1}} g_{F}\left(\mu_{f}+\omega\right)+\int_{\mathbf{P}^{1}} \log \left\|J_{F}\right\|_{0} \omega$.

(ii) $L(f)+\log d=\int_{\mathbf{P}^{1}} g_{F}\left[C_{f}\right]-2(d-1) \int_{\mathbf{P}^{1}} g_{F}\left(\mu_{f}+\omega\right)+\int_{\mathbf{C}^{2}} \log \left|\operatorname{det} F^{\prime}\right| m$.

(iii) If $\tilde{c}_{1}, \ldots, \tilde{c}_{2 d-2}$ are chosen such that $\operatorname{det} F^{\prime}(z)=\Pi_{j=1}^{2 d-2} \tilde{c}_{j} \wedge z$ one has:

$$
L(f)+\log d=\Sigma_{j} G_{F}\left(\tilde{c}_{j}\right)-(d-1)\left(1+2 \int_{\mathbf{P}^{1}} g_{F}\left(\mu_{f}+\omega\right)\right) .
$$

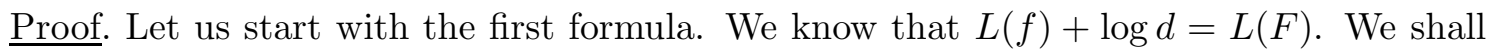
use the formalism introduced in the Subsection 1.5. By the first assertion of Lemma 1.4 and the definition of $\mu_{f}$ we have

$$
L(F)=\int_{\mathbf{P}^{1}} \log \left\|J_{F}\right\|_{G_{F}} \mu_{f}=\int_{\mathbf{P}^{1}} \log \left\|J_{F}\right\|_{G_{F}} d d^{c} g_{F}+\int_{\mathbf{P}^{1}} \log \left\|J_{F}\right\|_{G_{F}} \omega .
$$

After an integration by parts (see next section for a carefull justification) the identity (3.1) yields

$$
L(F)=\int_{\mathbf{P}^{1}} g_{F} d d^{c} \log \left\|J_{F}\right\|_{G_{F}}+\int_{\mathbf{P}^{1}} \log \left\|J_{F}\right\|_{G_{F}} \omega
$$


Using Poincaré-Lelong equation $d d^{c} \log \left\|J_{F}\right\|_{G_{F}}=-(2 d-2) \mu_{f}+\left[C_{f}\right]$, (3.2) becomes:

$$
L(F)=\int_{\mathbf{P}^{1}} g_{F}\left[C_{f}\right]-(2 d-2) \int_{\mathbf{P}^{1}} g_{F} \mu_{f}+\int_{\mathbf{P}^{1}} \log \left\|J_{F}\right\|_{G_{F}} \omega .
$$

After observing that $\|\cdot\|_{G_{F}}=e^{-(2 d-2) g_{F}}\|\cdot\|_{0}$ we may rewrite the last integral in (3.3) as: $\int_{\mathbf{P}^{1}} \log \left\|J_{F}\right\|_{0} \omega-(2 d-2) \int_{\mathbf{P}^{1}} g_{F} \omega$ and this gives our first formula.

In order to establish the second formula, we simply transform the first one by using the second assertion of Lemma 1.4.

Let us finally prove the third formula. Picking $U_{j} \in \mathbf{U}(2, \mathbf{C})$ such that $U_{j}^{-1}\left(\tilde{c}_{j}\right)=$ $\left(\left\|\tilde{c}_{j}\right\|, 0\right)$ we have $U_{j} z \wedge \tilde{c}_{j}=-z_{2}\left\|\tilde{c}_{j}\right\|$. Since $\int_{\mathbf{C}^{2}} \log \left|z_{2}\right| m=-\frac{1}{2}$, we get $\int_{\mathbf{C}^{2}} \log \left|\operatorname{det} F^{\prime}\right| m=$ $\Sigma_{j} \int_{\mathrm{C}^{2}} \log \left|U_{j} z \wedge \tilde{c}_{j}\right| m=\Sigma_{j} \log \left\|\tilde{c}_{j}\right\|-(d-1)$.

On the other hand, $\int_{\mathbf{P}^{1}} g_{F}\left[C_{f}\right]=\Sigma_{j} g_{F} \circ \pi\left(\tilde{c}_{j}\right)=\Sigma_{j} G_{F}\left(\tilde{c}_{j}\right)-\Sigma_{j} \log \left\|\left(\tilde{c}_{j}\right)\right\|$. It then suffices to report these identities in the second formula.

The usefullness of our formulas stays on the fact that the function $B(F):=\int_{\mathbf{P}^{1}} g_{F}\left(\mu_{f}+\omega\right)$ is pluriharmonic. This important property is easy to check by considering the formulas for both $f$ and $f^{2}$. Indeed, since $L\left(f^{2}\right)=2 L(f), G_{F^{2}}=G_{F}$ and (consequently) $B\left(F^{2}\right)=B(F)$ one immediately obtains a pluriharmonic expression for $B(F)$ by comparison.

Theorem 3.2 The function $B(F):=\int_{\mathbf{P}^{1}} g_{F}\left(\mu_{f}+\omega\right)$ is pluriharmonic on $\mathcal{H}_{d}\left(\mathbf{C}^{2}\right)$.

Using the third formula of Theorem 3.1 and Theorem 3.2 we obtain the following corollary previously obtained by De Marco (see 8 ). It allows to relate the pluriharmonicity of $L(F)$ with the stability of the dynamic of critical points. As we shall see in Section 5 this is a key point when approaching the Mane-Sad-Sullivan theory via potentialist methods.

Corollary 3.3 Let $\left\{f_{\lambda}\right\}_{\lambda \in X}$ be an holomorphic family of rational maps of degree $d$. Assume that the critical points of $f_{\lambda}$ are given by $\left\{c_{1}(\lambda), \ldots, c_{2 d-2}(\lambda)\right\}$ where the $c_{j}(\lambda)$ are holomorphic maps on $X$. Then $d d^{c} L\left(f_{\lambda}\right)=d d^{c} \sum_{j=1}^{2 d-2} G_{F}\left(\tilde{c}_{j}\right)$.

Using Proposition 1.2 (ii), one also reads on the third formula of Theorem 3.1 that the Lyapounov exponent $L(F)$ is an Hölder-continuous function in $F$. The continuity was first proved by Mane [15].

Corollary 3.4 The function $L(F)$ is p.s.h and Hölder-continuous on $\mathcal{H}_{d}\left(\mathbf{C}^{2}\right)$.

Proof of theorem [3.2. We may consider a local holomorphic parametrization $\lambda \mapsto F_{\lambda}$ of $\mathcal{H}_{d}\left(\mathbf{C}^{2}\right)$ defined on some open subset $U$ of $\mathbf{C}^{2 d+2}$. We shall denote $f_{\lambda}$ the induced map on $\mathbf{P}^{1}$ and set $B(\lambda):=B\left(F_{\lambda}\right)$. There exists an analytic subset $A$ of $U$ such that, for any $\lambda \in U \backslash A$, the critical points of $f_{\lambda}$ consist in $2 d-2$ distinct, regular values of $f_{\lambda}$. As the function $B(F)$ is locally bounded, it suffices to show that it is pluriharmonic on any 
sufficently small ball contained in $U \backslash A$.

On such a ball $B$, there are $2 d-2$ holomorphic maps $\tilde{c}_{j}$ such that

$$
\operatorname{det} F_{\lambda}^{\prime}=\Pi_{j=1}^{2 d-2} \quad \tilde{c}_{j}(\lambda) \wedge z .
$$

Moreover, for each $1 \leq j \leq 2 d-2$, there are $d$ holomorphic maps $\tilde{c}_{j, i}$ such that $F_{\lambda} \circ \tilde{c}_{j, i}(\lambda)=$ $\tilde{c}_{j}(\lambda)$ and therefore:

$$
\operatorname{det} F_{\lambda}^{2^{\prime}}=h(\lambda)\left(\Pi_{j=1}^{2 d-2} \Pi_{i=1}^{d} \quad \tilde{c}_{i, j}(\lambda) \wedge z\right)\left(\Pi_{j=1}^{2 d-2} \tilde{c}_{j}(\lambda) \wedge z\right)
$$

where $h$ is a non-vanishing holomorphic function on $B$. Let $N$ denote the degree of $\operatorname{det} F_{\lambda}^{2^{\prime}}$, after setting $\tilde{c}_{j, i}^{\prime}=h^{\frac{1}{N}} \tilde{c}_{j, i}$ and $\tilde{c}_{j}^{\prime}=h^{\frac{1}{N}} \tilde{c}_{j}$ we get

$$
\operatorname{det} F_{\lambda}^{2^{\prime}}=\left(\Pi_{j=1}^{2 d-2} \Pi_{i=1}^{d} \quad \tilde{c}_{i, j}^{\prime}(\lambda) \wedge z\right)\left(\Pi_{j=1}^{2 d-2} \quad \tilde{c}_{j}^{\prime}(\lambda) \wedge z\right)
$$

We are now in order to use the third formula of Theorem 3.1 for $f_{\lambda}^{2}$. Observing that $G_{F^{2}}=G_{F}, \mu_{f^{2}}=\mu_{f}$ and $B\left(F^{2}\right)=B(F)$ it yields:

$$
\begin{array}{r}
L\left(f_{\lambda}^{2}\right)+\log d^{2}+\left(d^{2}-1\right)\left(2 B\left(F_{\lambda}\right)+1\right)=\sum_{j=1}^{2 d-2} \sum_{i=1}^{d} G_{F}\left(\tilde{c}_{j, i}^{\prime}(\lambda)\right)+\Sigma_{j=1}^{2 d-2} G_{F}\left(\tilde{c}_{j}^{\prime}(\lambda)\right) \\
=\log |h(\lambda)|+\sum_{j=1}^{2 d-2} \sum_{i=1}^{d} G_{F}\left(\tilde{c}_{j, i}(\lambda)\right)+\Sigma_{j=1}^{2 d-2} G_{F}\left(\tilde{c}_{j}(\lambda)\right) \\
=\log |h(\lambda)|+\Sigma_{j=1}^{2 d-2} \frac{1}{d} \sum_{i=1}^{d} G_{F} \circ F\left(\tilde{c}_{j, i}(\lambda)\right)+\Sigma_{j=1}^{2 d-2} G_{F}\left(\tilde{c}_{j}(\lambda)\right) \\
=\log |h(\lambda)|+2 \sum_{j=1}^{2 d-2} G_{F}\left(\tilde{c}_{j}(\lambda)\right) .
\end{array}
$$

On the other hand,for $f_{\lambda}$, the same formula gives:

$$
L\left(f_{\lambda}^{2}\right)+\log d^{2}=2\left(L\left(f_{\lambda}\right)+\log d\right)=2 \Sigma_{j=1}^{2 d-2} G_{F}\left(\tilde{c}_{j}(\lambda)\right)-2(d-1)\left(2 B\left(F_{\lambda}\right)+1\right) .
$$

By comparison we thus obtain $2 B(\lambda)+1=\frac{1}{(d-1)^{2}} \log |h(\lambda)|$.

Remark 3.5 By using its pluriharmonicity, one may show that the function $B(F)$ is given by $B(F)=\frac{1}{d(d-1)} \log |\operatorname{Res}(F)|-\frac{1}{2}$. This gives again DeMarco's formula and will be proved in Proposition 4.9 in arbitrary dimension.

\section{A formula for the sum of Lyapunouv exponents of holo- morphic endomorphisms of $\mathbf{P}^{k}$}

Our aim here is to generalize the results of the previous section to endomorphisms of $\mathbf{P}^{k}$. We first establish a formula which relates the sum of Lyapounov exponents $L(f)$ with the Green current and the current of integration on the critical set. This extends Theorem 3.1 (i). We then generalize Theorem 3.2 and, in particular, obtain an intrinsic expression for $d d^{c} L$. 
Theorem 4.1 Let $f$ be an holomorphic endomorphism of $\mathbf{P}^{k}$ whose algebraic degree equals $d \geq 2$. Let $F$ be one of the lifts of $f$ to $\mathbf{C}^{k+1}$ and $T_{f}=d d^{c} g_{F}+\omega$ be the Greeen current of $f$. Then the sum of Lyapounov exponents $L(f)$ of $f$ is given by:

$$
L(f)+\log d=L(F)=H(F)-(k+1)(d-1) B(F)
$$

where

$$
H(F):=\sum_{j=0}^{k-1} \int_{C_{f}} g_{F} T_{f}^{j} \wedge \omega^{k-j-1}+\int_{\mathbf{P}^{k}} \log \left\|J_{F}\right\|_{0} \omega^{k}
$$

and

$$
B(F):=\sum_{j=0}^{k} \int_{\mathbf{P}^{k}} g_{F} T_{f}^{j} \wedge \omega^{k-j}
$$

Proof. According to Lemma 1.4 we have

$$
L(f)+\log d=\int_{\mathbf{P}^{k}} \log \left\|J_{F}\right\|_{G} T_{f}^{k}
$$

Let us start by showing that

$$
\begin{gathered}
L(f)+\log d=\sum_{j=0}^{k-1} \int_{\mathbf{P}^{k}} \log \left\|J_{F}\right\|_{G} d d^{c} g_{F} \wedge T_{f}^{j} \wedge \omega^{k-j-1}+ \\
\quad+\int_{\mathbf{P}^{k}} \log \left\|J_{F}\right\|_{G} \omega^{k}
\end{gathered}
$$

For this we first note that each term in the above sum is finite (this follows immediately from the Chern-Levine-Nirenberg inequalities) and then observe that:

$$
\begin{aligned}
d d^{c} g_{F} & \wedge\left(\sum_{j=0}^{k-1} T_{f}^{j} \wedge \omega^{k-j-1}\right)=\left(T_{f}-\omega\right) \wedge\left(\sum_{j=0}^{k-1} T_{f}^{j} \wedge \omega^{k-j-1}\right)= \\
= & \sum_{j=0}^{k-1} T_{f}^{j+1} \wedge \omega^{k-j-1}-\sum_{j=0}^{k-1} T_{f}^{j} \wedge \omega^{k-j}=T_{f}^{k}-\omega^{k} .
\end{aligned}
$$

We shall now use the following integration by part property which will be established separetely.

Fact:

$$
\begin{aligned}
A_{j} & :=\int_{\mathbf{P}^{k}} \log \left\|J_{F}\right\|_{G} d d^{c} g_{F} \wedge T_{f}^{j} \wedge \omega^{k-j-1}= \\
& =\int_{\mathbf{P}^{k}} g_{F} d d^{c} \log \left\|J_{F}\right\|_{G} \wedge T_{f}^{j} \wedge \omega^{k-j-1} .
\end{aligned}
$$

This allows us to transform the identity (4.4) and get:

$$
L(f)+\log d=\sum_{j=0}^{k-1} \int_{\mathbf{P}^{k}} g_{F} d d^{c} \log \left\|J_{F}\right\|_{G} \wedge T_{f}^{j} \wedge \omega^{k-j-1}+
$$




$$
+\int_{\mathbf{P}^{k}} \log \left\|J_{F}\right\|_{G} \omega^{k}
$$

Next, by the Poincaré-Lelong equation $d d^{c} \log \left\|J_{F}\right\|_{G}=\left[C_{f}\right]-(k+1)(d-1) T_{f}$, we obtain:

$$
\begin{gathered}
L(f)+\log d=\sum_{j=0}^{k-1} \int_{\mathbf{P}^{k}} g_{F} T_{f}^{j} \wedge \omega^{k-j-1} \wedge\left[C_{f}\right]- \\
-(k+1)(d-1) \sum_{j=0}^{k-1} \int_{\mathbf{P}^{k}} g_{F} T_{f}^{j+1} \wedge \omega^{k-j-1}+\int_{\mathbf{P}^{k}} \log \left\|J_{F}\right\|_{G} \omega^{k} .
\end{gathered}
$$

Finally, as \|\|$_{G}=e^{-(k+1)(d-1) g_{F}}\|\|_{0}$, we may replace the last integral in (4.6) by $\int_{\mathbf{P}^{k}} \log \left\|J_{F}\right\|_{0} \omega^{k}-(k+1)(d-1) \int_{\mathbf{P}^{k}} g_{F} \omega^{k}$ and this immediately yields to the expected formula.

It remains to establish the Fact. We shall proceed by regularization and use the following lemma.

Lemma 4.2 Let $\left\{\phi_{n}\right\}_{n \in \mathbf{N}^{*}}$ be a decreasing sequence of increasing smooth convex functions on $\mathbf{R}$ such that:

$$
\begin{aligned}
& \left.\left.\phi_{n}(x)=-n, \text { on }\right]-\infty,-n-\frac{1}{n}\right] \\
& \left.\left.\phi_{n}(x)=x, \text { on }\right]-n+\frac{1}{n},+\infty\right] \\
& 0 \leq \phi^{\prime}(x) \leq 1, \quad \forall x \in \mathbf{R} .
\end{aligned}
$$

Let $\log _{n}(x)$ be defined by $\log _{n}(x):=\phi_{n}(\log x)$. Then $\left\{\log _{n}\left\|J_{F}\right\|_{0}\right\}_{n \in \mathbf{N}^{*}}$ is a decreasing sequence of smooth functions, which converges to $\log \left\|J_{F}\right\|_{0}$. Moreover $d d^{c} \log _{n}\left\|J_{F}\right\|_{0}+$ $(k+1)(d-1) \omega \geq 0$ for all $n \in \mathbf{N}^{*}$.

The proof is by straightforward computation and we omit it.

Proof of the Fact. Let us first separate $A_{j}$ in two terms by using the relation \|\|$_{G_{F}}=e^{-(k+1)(d-1) g_{F}}\|\|_{0}$.

$$
\begin{gathered}
A_{j}=\int_{\mathbf{P}^{k}} \log \left\|J_{F}\right\|_{0} d d^{c} g_{F} \wedge T_{f}^{j} \wedge \omega^{k-j-1}- \\
-(k+1)(d-1) \int_{\mathbf{P}^{k}} g_{F} d d^{c} g_{F} \wedge T_{f}^{k} \wedge \omega^{k-j-1}
\end{gathered}
$$

We will regularize and integrate by part each term separately. Let us start with the second term $A_{j, 2}$. As $g_{F, n}$ is uniformely converging to $g_{F}$ we have

$$
A_{j, 2}=\lim _{n \rightarrow \infty} \int_{\mathbf{P}^{k}} g_{F, n} d d^{c} g_{F} \wedge T_{f}^{j} \wedge \omega^{k-j-1}
$$

then, by Stokes Theorem and again by uniform convergence,

$$
\begin{aligned}
A_{j, 2}= & \lim _{n \rightarrow \infty} \int_{\mathbf{P}^{k}} g_{F} d d^{c} g_{F, n} \wedge T_{f}^{j} \wedge \omega^{k-j-1}= \\
& =\int_{\mathbf{P}^{k}} g_{F} d d^{c} g_{F} \wedge T_{f}^{j} \wedge \omega^{k-j-1} .
\end{aligned}
$$


We now treat the first term $A_{j, 1}$. By the Lemma 4.2 $\log _{n}\left\|J_{F}\right\|_{0}$ is decreasingly converging to $\log \left\|J_{F}\right\|_{0}$ and $d d^{c} \log _{n}\left\|J_{F}\right\|_{0}+(k+1)(d-1) \omega \geq 0$ for all $n \in \mathbf{N}^{*}$. We may thus use monotone convergence theorem and Stokes.

$$
\begin{gathered}
A_{j, 1}=\lim _{n \rightarrow \infty} \int_{\mathbf{P}^{k}} \log _{n}\left\|J_{F}\right\|_{0} d d^{c} g_{F} \wedge T_{f}^{j} \wedge \omega^{k-j-1}= \\
=\lim _{n \rightarrow \infty} \int_{\mathbf{P}^{k}} g_{F} d d^{c} \log _{n}\left\|J_{F}\right\|_{0} \wedge d d^{c} g_{F} \wedge T_{f}^{j} \wedge \omega^{k-j-1}= \\
=\int_{\mathbf{P}^{k}} g_{F} d d^{c} \log \left\|J_{F}\right\|_{0} \wedge d d^{c} g_{F} \wedge T_{f}^{j} \wedge \omega^{k-j-1}
\end{gathered}
$$

Our aim now is to compute $d d^{c} L\left(f_{\lambda}\right)$ when $\left\{f_{\lambda}\right\}_{\lambda \in X}$ is an holomorphic family of endomorphisms of $\mathbf{P}^{k}$. We need the following technical Proposition which will be established in the Appendix.

Proposition 4.3 Let $X^{m} \stackrel{\pi}{\longrightarrow} Y^{n}$ be an holomorphic submersion between complex manifolds. If $R$ is a current on $X$, for $y \in Y$ the slice (if it exists) of $R$ along the fiber $\pi^{-1}(y)$ is denoted by $R_{y}$. Let $u_{1}, \ldots, u_{h}$ be almost plurisubharmonic, locally bounded functions on $X$ and $T$ be a positive, closed $(k, k)$ current on $X$, with $h+k \leq m-n$. Thus, for a.e. $y \in Y$,

$$
\begin{gathered}
\left(u_{1} d d^{c} u_{2} \wedge \cdots \wedge d d^{c} u_{h} \wedge T\right)_{y}= \\
=u_{1 \mid \pi^{-1}(y)} d d^{c}\left(u_{2 \mid \pi^{-1}(y)}\right) \wedge \cdots \wedge d d^{c}\left(u_{h \mid \pi^{-1}(y)}\right) \wedge T_{y} .
\end{gathered}
$$

Let us recall that, for a $(k, k)$-current $R$ on $X$, slicing is characterised (for a.e. $y \in Y$ ) by the following identity:

$$
\int_{X} R \wedge \psi \wedge \pi^{*} \phi=\int_{Y}\left(\int_{\pi^{-1}(y)} R_{y} \wedge \iota_{y}^{*} \psi\right) \phi
$$

for every smooth $(n, n)$-form $\phi$ on $Y$ and for every smooth and compactely supported $(m-n-k, m-n-k)$-form $\psi$ on $X$ (here $\iota_{y}: \pi^{-1}(y) \rightarrow X$ is the inclusion.)

By Theorem $4.1 L\left(f_{\lambda}\right)=H\left(f_{\lambda}\right)-(k+1)(d-1) B\left(f_{\lambda}\right)$. We first compute $d d^{c} H$ :

Proposition 4.4 Let $\left\{f_{\lambda}\right\}_{\lambda \in X}$ be an holomorphic family of endomorphisms of $\mathbf{P}^{k}$ such that there is an holomorphic lift $\left\{F_{\lambda}\right\}_{\lambda \in X}$ to $\mathbf{C}^{k+1}$. Then

$$
d d^{c} H\left(F_{\lambda}\right)=p_{*}\left(\left(d d^{c} g_{F_{\lambda}}+\omega\right)^{k} \wedge\left[C_{X}\right]\right)
$$

where $C_{X}:=\left\{(\lambda, z) \in X \times \mathbf{P}^{k} ; z \in C_{f_{\lambda}}\right\}$ and $p: X \times \mathbf{P}^{k} \rightarrow X$ is the canonical projection. 
Remark. $d d^{c} g_{F_{\lambda}}$ involves derivatives in both $\lambda \in X$ and $z \in \mathbf{P}^{k}$.

Proof. Let $q=\operatorname{dim}_{\mathbf{C}} X$, for a $(q-1, q-1)$-form $\phi$ with compact support on $X$ we have

$$
\begin{aligned}
<d d^{c} H, \phi>=\int_{X}( & \left.\int_{\mathbf{P}^{k}} g_{F_{\lambda}}\left(\sum_{j=0}^{k-1}\left(d d^{c} g_{F_{\lambda}}+\omega\right)^{j} \wedge \omega^{k-j-1}\right) \wedge\left[C_{f_{\lambda}}\right]\right) d d^{c} \phi+ \\
& +\int_{X}\left(\int_{\mathbf{P}^{k}} \log \left\|J_{F_{\lambda}}\right\|_{0} \omega^{k}\right) d d^{c} \phi .
\end{aligned}
$$

Since $\left[C_{f_{\lambda}}\right]$ is the slice of $\left[C_{X}\right]$ (see [28] (10.4)), by means of the Proposition 4.3 the first integral is

$$
\int_{X \times \mathbf{P}^{k}} p^{*} \phi \wedge d d^{c} g_{F_{\lambda}} \wedge\left(\sum_{j=0}^{k-1}\left(d d^{c} g_{F_{\lambda}}+\omega\right)^{j} \wedge \omega^{k-j-1}\right) \wedge\left[C_{X}\right] .
$$

On Poincaré - Lelong formula $\left[C_{X}\right]=d d^{c} \log \left\|J_{F_{\lambda}}\right\|_{0}+(k+1)(d-1) \omega$ one sees that $\log \left\|J_{F_{\lambda}}\right\|_{0}$ is almost plurisubharmonic and therefore locally sommable. Thus the second integral is

$$
\begin{gathered}
\int_{X \times \mathbf{P}^{k}} p^{*} \phi \wedge d d^{c} \log \left\|J_{F_{\lambda}}\right\|_{0} \wedge \omega^{k}= \\
=\int_{X \times \mathbf{P}^{k}} p^{*} \phi \wedge \omega^{k} \wedge\left[C_{X}\right]-(k+1)(d-1) \int_{X \times \mathbf{P}^{k}} p^{*} \phi \wedge \omega^{k+1} .
\end{gathered}
$$

But $\omega^{k+1}=0$ on $X \times \mathbf{P}^{k}$ and therefore, after summing up, we obtain:

$$
<d d^{c} H, \phi>=\int_{C_{X}} p^{*} \phi \wedge\left(d d^{c} g_{F_{\lambda}}+\omega\right)^{k}=<\left(d d^{c} g_{F_{\lambda}}+\omega\right)^{k} \wedge\left[C_{X}\right], p^{*} \phi>.
$$

Now we can also extend Theorem 3.2 to the $k$-dimensional case. We shall use the same device, that is to compare formulas for $F_{\lambda}$ and $F_{\lambda}^{2}$.

Theorem 4.5 The function $B(F)$ is pluriharmonic on $\mathcal{H}_{d}\left(\mathbf{C}^{k+1}\right)$.

Proof. Let us start with a:

Claim: $H(F)$ is p.s.h. on $\mathcal{H}_{d}\left(\mathbf{C}^{k+1}\right)$ and $d d^{c} H(F)=2 d d^{c} H\left(F^{2}\right)$.

Proof of the Claim. Let $X:=\mathcal{H}_{d}\left(\mathbf{C}^{k+1}\right)$, the projection $X \ni F \mapsto f \in \mathcal{H}_{d}\left(\mathbf{P}^{k}\right)$ defines an holomorphic family $\{f\}_{F \in X}$ and the plurisubharmonicity follows from Proposition 4.4

Set $C_{X}:=\left\{(F, z) \in X \times \mathbf{P}^{k} ; z \in C_{f}\right\}$ and $C_{X}^{\prime}:=\left\{(F, z) \in X \times \mathbf{P}^{k} ; z \in C_{f_{\lambda}^{2}}\right\}$. As $F^{2}$ and $F$ have the same Green function $g_{F}$, Proposition 4.4 gives

$$
d d^{c} H\left(F^{2}\right)=p_{*}\left(\left(d d^{c} g_{F}+\omega\right)^{k} \wedge\left[C_{X}^{\prime}\right]\right) .
$$

Consider the map $\Phi: X \times \mathbf{P}^{k} \rightarrow X \times \mathbf{P}^{k}$ defined by $\Phi(F, z):=(F, f(z))$ and observe that $C_{X}^{\prime}=\Phi^{-1}\left(C_{X}\right) \cup C_{X}$; thus

$$
d d^{c} H\left(F^{2}\right)=p_{*}\left(\left(d d^{c} g_{F}+\omega\right)^{k} \wedge\left[\Phi^{-1}\left(C_{X}\right)\right]\right)+p_{*}\left(\left(d d^{c} g_{F}+\omega\right)^{k} \wedge\left[C_{X}\right]\right) .
$$


From $G_{F} \circ F=d . G_{F}$, it follows $\Phi^{*}\left(d d^{c} g_{F}+\omega\right)=d \cdot\left(d d^{c} g_{F}+\omega\right)$, thus

$$
d d^{c} H\left(F^{2}\right)=p_{*}\left(\frac{1}{d^{k}} \Phi^{*}\left(d d^{c} g_{F}+\omega\right)^{k} \wedge\left[\Phi^{-1}\left(C_{X}\right)\right]\right)+d d^{c} H(F) .
$$

Since $\Phi: \Phi^{-1}\left(C_{X}\right) \rightarrow C_{X}$ has topological degree $d^{k}$, we have $\Phi_{*}\left[\Phi^{-1}\left(C_{X}\right)\right]=d^{k}\left[C_{X}\right]$ and thus:

$$
d d^{c} H\left(F^{2}\right)=p_{*}\left(\frac{1}{d^{k}}\left(d d^{c} g_{F}+\omega\right)^{k} \wedge \Phi_{*}\left[\Phi^{-1}\left(C_{X}\right)\right]\right)+d d^{c} H(F)=2 d d^{c} H(F) .
$$

End of the proof of Theorem 4.5] Since $L\left(F^{2}\right)=2 L(F)$ we have

$$
d d^{c} L\left(F^{2}\right)=2 d d^{c} L(F)=2\left(d d^{c} H(F)-(k+1)(d-1) d d^{c} B(F)\right)
$$

on the other hand, since $B\left(F^{2}\right)=B(F)$, we may use the Claim and get:

$$
\begin{gathered}
d d^{c} L\left(F^{2}\right)=d d^{c} H\left(F^{2}\right)-(k+1)\left(d^{2}-1\right) d d^{c} B\left(F^{2}\right)= \\
=2 d d^{c} H(F)-(k+1)\left(d^{2}-1\right) d d^{c} B(F) .
\end{gathered}
$$

By comparison we get $(d-1)^{2} d d^{c} B(f)=0$, thus $B$ is pluriharmonic on $\mathcal{H}_{d}\left(\mathbf{C}^{k+1}\right)$.

Corollary 4.6 Let $\left\{f_{\lambda}\right\}_{\lambda \in X}$ be an holomorphic family of endomorphisms of $\mathbf{P}^{k}$ with algebraic degree $d \geq 2$. Then

$$
d d^{c} L\left(f_{\lambda}\right)=p_{*}\left(\left(d d^{c} g_{F_{\lambda}}+\omega\right)^{k} \wedge\left[C_{X}\right]\right)
$$

and on $X \times \mathbf{P}^{k}$

$$
\left(d d^{c} g_{F_{\lambda}}+\omega\right)^{k+1}=0
$$

Remark 4.7 As we have already noted, the operator $d d^{c}$ in the above formula involves derivatives in both $\lambda \in X$ and $z \in \mathbf{P}^{k}$; thus the current $\tilde{T}:=d d^{c} g_{F_{\lambda}}+\omega$ is different from the Green current. This current depends only on the family $\left\{f_{\lambda}\right\}$ and not on the local lift $\left\{F_{\lambda}\right\}$; moreover it is positive on $X \times \mathbf{P}^{k}$ since $G_{F_{\lambda}}(z)$ is p.s.h. on $X \times\left(\mathbf{C}^{k+1} \backslash\{0\}\right.$ ) (see Proposition [1.2). Using the current $\tilde{T}$ we may express the formulas of Corollary 4.6 in a synthetic way and avoid any reference to the lift $\left\{F_{\lambda}\right\}$ which in general is only defined locally:

$$
d d^{c} L\left(f_{\lambda}\right)=p_{*}\left(\tilde{T}^{k} \wedge\left[C_{X}\right]\right)
$$

and

$$
\tilde{T}^{k+1}=0 .
$$


Proof of Corollary [4.6. From Proposition 4.4 and Theorem 4.5 we get the first statement.

We argue as in the proof of the Proposition 4.4 (using again Proposition 4.3) and choosing an open subset $V \subset X$ such that there is an holomorphic family of lifts $\left\{F_{\lambda}\right\}_{\lambda \in V}$ : we have

$$
\begin{gathered}
<d d^{c} B, \phi>=\int_{V}\left(\int_{\mathbf{P}^{k}} g_{F_{\lambda}}\left(\sum_{j=0}^{k}\left(d d^{c} g_{F_{\lambda}}+\omega\right)^{j} \wedge \omega^{k-j}\right)\right) d d^{c} \phi= \\
=\int_{V \times \mathbf{P}^{k}} p^{*}(\phi) \wedge d d^{c} g_{F_{\lambda}} \wedge\left(\sum_{j=0}^{k}\left(d d^{c} g_{F_{\lambda}}+\omega\right)^{j} \wedge \omega^{k-j}\right)
\end{gathered}
$$

Then, as in (4.5) we get

$$
<d d^{c} B, \phi>=\int_{V \times \mathbf{P}^{k}} p^{*}(\phi) \wedge\left(\tilde{T}^{k+1}-\omega^{k+1}\right)=<p_{*}\left(\tilde{T}^{k+1}\right), \phi>,
$$

because $\omega^{k+1}$ vanish. Finally, since $d d^{c} B=0$ and $\tilde{T}$ is positive, we get $\tilde{T}^{k+1}=0$

By Theorem 4.5. the function $B$ is pluriharmonic: this suggests the existence of a simpler analytic expression for $B$, as indeed Proposition 4.9 states. Since $B$ is defined by means of dynamical quantities this result seems of some interest. We shall need the following lemma.

Lemma 4.8 $H^{1}\left(\mathcal{H}_{d}\left(\mathbf{P}^{k}\right) ; \mathbf{R}\right)=0$

Proof. The Fubini-Study form $\omega$ generates $H^{2 N-2}\left(\mathbf{P}^{N} ; \mathbf{R}\right)$ and $\left(\iota^{*}\left(\omega^{N-1}\right), \Sigma_{d}\right)=\int_{\Sigma_{d}} \omega^{N-1}=$ $\operatorname{vol}\left(\Sigma_{d}\right) \neq 0$, where $\iota: \Sigma_{d} \rightarrow \mathbf{P}^{N}$ is the inclusion; therefore the map $\mathbf{R}=H^{2 N-2}\left(\mathbf{P}^{N} ; \mathbf{R}\right) \stackrel{\iota^{*}}{\rightarrow}$ $H^{2 N-2}\left(\Sigma_{d} ; \mathbf{R}\right)=\mathbf{R}$ is an isomorphism. Hence, from the exact sequence

$$
H^{2 N-2}\left(\mathbf{P}^{N} ; \mathbf{R}\right) \stackrel{\iota^{*}}{\rightarrow} H^{2 N-2}\left(\Sigma_{d} ; \mathbf{R}\right) \rightarrow H^{2 N-1}\left(\mathbf{P}^{N}, \Sigma_{d} ; \mathbf{R}\right) \rightarrow H^{2 N-1}\left(\mathbf{P}^{N} ; \mathbf{R}\right)=0,
$$

it follows that

$$
H^{2 N-1}\left(\mathbf{P}^{N}, \Sigma_{d} ; \mathbf{R}\right)=0 .
$$

Observe that $\Sigma_{d}$ is an euclidean neighbourhood retract (see [1] Prop. IV.8.2, VIII.6.12, VIII.7.2) thus $H^{j}\left(\mathbf{P}^{N}, \Sigma_{d} ; \mathbf{R}\right)=H_{2 N-j}\left(\mathbf{P}^{N} \backslash \Sigma_{d} ; \mathbf{R}\right) . \quad$ In particular $0=H^{2 N-1}\left(\mathbf{P}^{N}, \Sigma_{d} ; \mathbf{R}\right)=H_{1}\left(\mathbf{P}^{N} \backslash \Sigma_{d} ; \mathbf{R}\right)$ and then also its dual $H^{1}\left(\mathbf{P}^{N} \backslash \Sigma_{d} ; \mathbf{R}\right)$ vanishes.

Now we can establish:

Proposition 4.9 There exists a constant $C_{d, k}$ such that, $\forall F \in \mathcal{H}_{d}\left(\mathbf{C}^{k+1}\right)$,

$$
B(F)=\frac{1}{d^{k}(d-1)} \log |\operatorname{Res}(F)|+C_{d, k}
$$




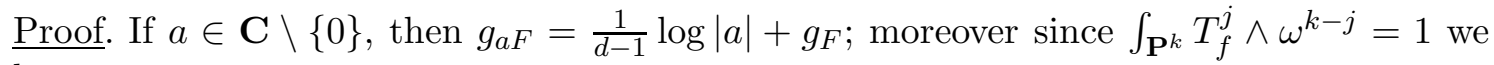
have

$$
B(a F)=\frac{(k+1)}{d-1} \log |a|+B(F) .
$$

The polynomial $\operatorname{Res}(F)$ is homogeneous of degree $(k+1) d^{k}$, thus the function $d^{k}(d-1) B(F)-\log |\operatorname{Res}(F)|$ is homogeneous of degree 0 and defines a pluriharmonic function $\Phi: \mathcal{H}_{d}\left(\mathbf{P}^{k}\right) \rightarrow \mathbf{R}$ such that

$$
\forall F \in \mathcal{H}_{d}\left(\mathbf{C}^{k+1}\right), \quad \Phi \circ \pi(F)=d^{k}(d-1) B(F)-\log |\operatorname{Res}(F)| .
$$

Let $\mathcal{P H}$ be the sheaf of pluriharmonic functions, by means of Lemma 4.8. from the exact sequence $0 \rightarrow \mathbf{R} \stackrel{i}{\rightarrow} \mathcal{O} \stackrel{R e}{\rightarrow} \mathcal{P} \mathcal{H} \rightarrow 0$ we get that $H^{0}\left(\mathcal{H}_{d}\left(\mathbf{P}^{k}\right), \mathcal{O}\right) \stackrel{R e}{\rightarrow} H^{0}\left(\mathcal{H}_{d}\left(\mathbf{P}^{k}\right), \mathcal{P} \mathcal{H}\right)$ is surjective; therefore there exists a holomorphic function $\varphi$ on $\mathcal{H}_{d}\left(\mathbf{P}^{k}\right)$ such that $\operatorname{Re}(\varphi)=\Phi$. Setting $\psi:=e^{\varphi}$, we obtain

$$
\log |\psi|=\Phi \text { on } \mathcal{H}_{d}\left(\mathbf{P}^{k}\right) .
$$

Using Proposition 1.2 one sees that $B$ is bounded from above on $K \cap \mathcal{H}_{d}\left(\mathbf{P}^{k}\right)$, for every compact $K \subset \mathbf{C}^{N+1}$. It follows that $\operatorname{Res}(F) \cdot \psi(\pi(F))$ is locally bounded and thus can be extended to an holomorphic function $\chi$ on $\mathbf{C}^{N+1}$. But $\chi$ is clearly homogeneous with the same degree that $\operatorname{Res}(F)$, thus $\chi$ is a polynomial on $\mathbf{C}^{N+1}$ and $\psi$ is a constant.

\section{Proposition 4.10}

$$
C_{d, k}=-\frac{1}{2} \sum_{j=1}^{k}\left(\frac{1}{(d-1)^{k-j}} \sum_{s=1}^{j} \frac{1}{s}\right)
$$

Proof. See Appendix.

Remark 4.11 In the 1-dimensional case Propositions 4.9 and 4.10 give an independent proof of DeMarco's formula (see [8] Corollary 1.6) since

$$
\int_{\mathbf{P}^{1}} g_{F}\left(\mu_{f}+\omega\right)=\frac{1}{2}(\log |\operatorname{Res}(F)|-1) .
$$

\section{The bifurcation currents.}

In this section, we associate to any holomorphic family $\left\{f_{\lambda}\right\}_{\lambda \in X}$ in $\mathcal{H}_{d}\left(\mathbf{P}^{1}\right)$ a collection of bifurcation currents $\left(d d^{c} L\left(f_{\lambda}\right)\right)^{p}$ where $1 \leq p \leq \operatorname{dim}_{\mathbf{C}} X$. Our main goal is to give a rather precise description of the supports and, more precisely, to compare them with the hypersurfaces consisting of mappings having neutral cycles. The extremal cases $p=1$ and $p=2 d-2$ are of special interest. For $p=1$, we partially recover Mane-Sad-Sullivan work. For $p=2 d-2$, our description will become significant in the last section when introducing a bifurcation measure on the moduli space $\mathcal{M}_{d}$. Let us underline that we shall proceed by induction on $p$.

In order to state the results we must precise a few notations. 
Definition 5.1 We will consider an holomorphic family $\left\{f_{\lambda}\right\}_{\lambda \in X}$ of elements of $\mathcal{H}_{d}\left(\mathbf{P}^{1}\right)$ parametrized over an arbitrary complex manifold $X$. We set $D:=2 d-2$ and denote by $L(\lambda)$ the p.s.h. function on $X$ defined by $L(\lambda):=L\left(f_{\lambda}\right)$. Next we introduce the following subsets of $X$.

$\mathcal{R}:=\left\{\lambda_{0} \in X /\right.$ the repulsive cycles of sufficently high period of $f_{\lambda}$ move holomorphically on a fixed neighbourhood $U_{0}$ of $\left.\lambda_{0}\right\}$

$\mathcal{S}:=\left\{\lambda_{0} \in X / \lambda \rightarrow f_{\lambda}^{n}\left(C_{\lambda}\right)\right.$ is equicontinous at $\left.\lambda_{0}\right\}$

$\operatorname{Per}\left(X, n, e^{2 i \pi \theta}\right):=\left\{\lambda_{0} \in X / f_{\lambda_{0}}\right.$ has a cycle of period $n$ and multiplier $\left.e^{2 i \pi \theta}\right\}$

It may happen that $\operatorname{Per}\left(X, n, e^{2 i \pi \theta}\right)$ is empty or coincides with $X$. However, if it is not empty and if $e^{2 i \pi \theta} \neq 1$, then $\operatorname{Per}\left(X, n, e^{2 i \pi \theta}\right)$ is an hypersurface in $X$. In this situation we adopt the following convention: $\operatorname{Per}_{1}\left(X, n, e^{2 i \pi \theta}\right)$ always refers to some co-dimension one irreducible component of $\operatorname{Per}\left(X, n, e^{2 i \pi \theta}\right)$. For any dense subset $E$ of $] 0,1[$, we set

$$
\mathcal{Z}_{1}(X, E)=\bigcup_{n \in \mathbf{N}^{*}, \theta \in E} \operatorname{Per}_{1}\left(X, n, e^{2 i \pi \theta}\right)
$$

Let us recall that the set $\mathcal{R}$ has been implicitely considered in Theorem 2.2 which may be stated as $\mathcal{R} \cap S u p p\left(d d^{c} L\right)=\emptyset$. Note also that, in the definition of $\mathcal{S}$, the maps $\lambda \rightarrow f_{\lambda}^{n}\left(C_{\lambda}\right)$ are viewed as finitely valued holomorphic maps from $X$ to $\mathbf{P}^{1}$.

Our descritpion of $\operatorname{Supp}\left(d d^{c} L\right)$ contains a substantial part of Mane-Sad- Sullivan theory (see [16]). The originality here relies on the potentialist nature of our proof.

Theorem 5.2 Let $E$ be a dense subset of $] 0,1\left[\right.$. Let $\left\{f_{\lambda}\right\}_{\lambda \in X}$ be an holomorphic family of rational maps of degree $d$ on $\mathbf{P}^{1}$. Then

$$
\overline{\mathcal{Z}}_{1}(X, E)=\mathcal{R}^{c}=\operatorname{Supp}\left(d d^{c} L\right)=\mathcal{S}^{c}
$$

Let us briefly sketch the proof before entering into details. The inclusion $\mathcal{S}^{c} \subset$ $\operatorname{Supp}\left(d d^{c} L\right)$ is a consequence of Corollary 3.3 and was already observed by DeMarco ([8], Theorem 1.1). The inclusion $\operatorname{Supp}\left(d d^{c} L\right) \subset \mathcal{R}^{c}$ was proved in Theorem 2.2] we recall that the main ingredient was the equidistribution of repulsive cycles. The inclusions $\mathcal{R}^{c} \subset \overline{\mathcal{Z}}_{1}(X, E) \subset \mathcal{S}^{c}$ are classical since Mane-Mad-Sullivan work. Their proofs, which we reproduce here for sake of completeness, only use the elementary fact that any attractive basin contains at least a critical point.

$\mathcal{S}^{c} \subset S u p p\left(d d^{c} L\right)$ : Let $\Omega$ be an open ball in $X$ on which $L$ is pluriharmonic; we have to show that $\Omega \subset \mathcal{S}$. There exists an analytic subset $A$ of $\Omega$ such that the $D$-valued holomorphic map $\lambda \mapsto C_{f_{\lambda}}$ is given by $C_{f_{\lambda}}=\left\{c_{1}(\lambda), \ldots, c_{D}(\lambda)\right\}$ where the $c_{j}(\lambda)$ are holomorphic maps around any point $\lambda_{0} \in \Omega \backslash A$. Shrinking $\Omega$ if necessary, we find a $D$-valued holomorphic map $\lambda \mapsto \tilde{C}_{f_{\lambda}}$ from $\Omega$ to $\mathbf{C}^{2} \backslash\{0\}$ which is associated to the same analytic set $A$ and such that $\pi \circ \tilde{C}_{f_{\lambda}}=C_{f_{\lambda}}$.

The product $\Pi\left(z \wedge \tilde{c}_{j}(\lambda)\right)$ is a well defined $D$-homogeneous polynomial on $\mathbf{C}^{2}$ whose coefficients are bounded holomorphic functions on $\Omega \backslash A$. It therefore coincides with the restriction of a polynomial $H$ with holomorphic coefficients on $\Omega$. Moreover, as $H$ is obviously proportional to $\operatorname{det} F_{\lambda}^{\prime}$, there exists a non-vanishing holomorphic function $\varphi$ on $\Omega$ such that $H=\varphi(\lambda) \operatorname{det} F_{\lambda}^{\prime}$. Thus, after replacing $\tilde{c}_{j}(\lambda)$ by $(\varphi(\lambda))^{1 / D} \tilde{c}_{j}(\lambda)$, we may assume that

$$
H=\Pi\left(z \wedge \tilde{c}_{j}(\lambda)\right)=\operatorname{det} F_{\lambda}^{\prime} ; \forall z \in \mathbf{C}^{2}, \forall \lambda \in \Omega
$$


In the same way, we may construct a sequence of $D$-homogeneous polynomials $H_{n}$ of the form

$$
H_{n}:=h(\lambda)^{-d^{n}} \Pi\left(z \wedge F_{\lambda}^{n}\left(\tilde{c}_{j}(\lambda)\right)\right)
$$

where $h$ is a non-vanishing holomorphic function on $\Omega$. We will see that for a good choice of $h$ the coefficents of $H_{n}$ are uniformly bounded holomorphic functions on $\Omega$. As $\pi\left(\left\{H_{n}(\lambda,)=0.\right\}\right)=f_{\lambda}^{n}\left(C_{f_{\lambda}}\right)$, this implies that $\Omega \subset \mathcal{S}$.

It remains to construct $h$. Since $d d^{c} L=0$ on $\Omega$, it follows from (5.1) and Corollary 3.3 that the function $\sum_{j=1}^{D} G_{\lambda}\left(\tilde{c}_{j}(\lambda)\right)$ is pluriharmonic on $\Omega \backslash A$. As it is continuous on $\Omega$, it is actually pluriharmonic on $\Omega$ and therefore coincides with $\log |h(\lambda)|$ for some non-vanishing holomorphic function $h$.

Let us now consider an arbitrary small ball $\omega$ contained in $\Omega \backslash A$. We will show that, for all $\lambda \in \omega$, one has $H_{n}(\lambda, z)=e^{-i d^{n} \theta_{\omega}} \Pi\left(z \wedge A_{j}(\lambda)\right)$ where $\theta_{\omega} \in \mathbf{R}$ and $A_{j}(\lambda) \in\left\{G_{\lambda}=\right.$ $0\}$. The conclusion will follow since $\cup_{\lambda \in \Omega}\left\{G_{\lambda}=0\right\} \subset \subset \mathbf{C}^{2}$. As each term in the sum $\sum_{j=1}^{D} G_{\lambda}\left(\tilde{c}_{j}(\lambda)\right)$ is p.s.h. on $\omega$, there are $D$ non-vanishing holomorphic functions $h_{j}$ such that $G_{\lambda}\left(\tilde{c}_{j}(\lambda)\right)=\log \left|h_{j}\right|$. Thus $\log |h|=\log \Pi\left|h_{j}\right|$ and $h=e^{i \theta_{\omega}} \Pi h_{j}$ for some $\theta_{\omega} \in \mathbf{R}$. Then, for any $\lambda \in \omega$, we get from (5.2):

$$
H_{n}(\lambda, z)=e^{-i d^{n} \theta_{\omega}} \Pi\left(h_{j}(\lambda)^{-d^{n}} z \wedge F_{\lambda}^{n}\left(\tilde{c}_{j}(\lambda)\right)=e^{-i d^{n} \theta_{\omega}} \Pi\left(z \wedge F_{\lambda}^{n}\left(\frac{\tilde{c}_{j}(\lambda)}{h_{j}(\lambda)}\right)\right) .\right.
$$

It finally suffices to set $A_{j}(\lambda):=\frac{\tilde{c}_{j}(\lambda)}{h_{j}(\lambda)}$ since, as desidered, we have $G_{\lambda}\left(A_{j}(\lambda)\right)=G_{\lambda}\left(\tilde{c}_{j}(\lambda)\right)-$ $\log \left|h_{j}(\lambda)\right|=0$.

$\operatorname{Supp}\left(d d^{c} L\right) \subset \mathcal{R}^{c}$ : this is given by Theorem 2.2

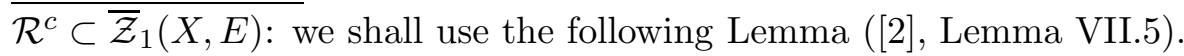

Lemma 5.3 Let $z_{0} \in \mathbf{P}^{1}$ be a repulsive fixed point of $f_{\lambda_{0}}^{n_{0}}\left(n_{0}\right.$ being the period of the associated cycle) and $B$ be a ball centered at $\lambda_{0}$ in $X$. Let $z(\lambda)$ be an holomorphic map defined on some neighbourhood of $\lambda_{0}$ in $X$ such that $z\left(\lambda_{0}\right)=z_{0}$ and $z(\lambda)$ is a repulsive fixed point of $f_{\lambda}^{n_{0}}$ (the points $z(\lambda)$ are given by the implicit function theorem). Then: either

i) $z(\lambda)$ holomorphically extends to $B$ and $z(\lambda)$ is a repulsive fixed point of $f_{\lambda}^{n_{0}}$ which belongs to a cycle of period $n_{0}$

or

ii) $z(\lambda)$ holomorphically extends along some path $\gamma$ joinging $\lambda_{0}$ to $\lambda_{1}$ in $B$ and $z\left(\lambda_{1}\right)$ is an attracting fixed point of $f_{\lambda_{1}}^{n_{0}}$. In particular, there are infinitely many values of $\lambda^{\prime}$ such that $z\left(\lambda^{\prime}\right)$ is a neutral fixed point of $f_{\lambda^{\prime}}^{n_{0}}$ and the corresponding multiplier contains an open subset of $S 1$. Again every $z(\lambda)$ belongs to a cycle of period $n_{0}$.

If $\lambda_{0} \in \mathcal{R}^{c}$ then, using the above lemma, we may find a non stationary sequence $\lambda_{k} \rightarrow \lambda_{0}$ such that $f_{\lambda_{k}}$ has a neutral cycle of period $n_{k}$ and a multiplier $e^{2 i \pi \theta_{k}}$ with $\theta_{k} \in E$. Since by Fatou theorem $f_{\lambda_{0}}$ has at most $6 d-6$ non-repulsive cycles, all but a finite number of $\operatorname{Per}\left(X, n_{k}, e^{2 i \pi \theta_{k}}\right)$ differ from $X$, this shows that $\lambda_{0} \in \overline{\mathcal{Z}}_{1}(X, E)$. 
$\overline{\mathcal{Z}}_{1}(X, E) \subset \mathcal{S}^{c}$ : we proceed by contradiction. Let $\lambda_{0} \in \mathcal{Z}_{1}(X, E)$ and $B$ an open ball in $\overline{X \text { such that } \lambda_{0}} \in B \subset \mathcal{S}$. Let $n_{0} \in \mathbf{N}^{*}$ and $\theta_{0} \in E$ such that $B \cap P e r_{1}\left(X, n_{0}, e^{2 i \pi \theta_{0}}\right) \neq \emptyset$. On a small ball $B^{\prime} \subset B$ centered at some point $\lambda_{1} \in P e r_{1}\left(X, n_{0}, e^{2 i \pi \theta_{0}}\right)$ there exists an holomorphic map $z(\lambda)$ such that $f_{\lambda}^{n_{0}}(z(\lambda))=z(\lambda)$. Moreover, as the multiplier of $f_{\lambda}^{n_{0}}$ at $z(\lambda)$ is not constant near $\lambda_{1}$ (otherwise $\operatorname{Per}_{1}\left(X, n_{0}, e^{2 i \pi \theta_{0}}\right)$ would coincide with $X$ ), we may find $\lambda_{2}, \lambda_{3} \in B^{\prime}$ such that $z\left(\lambda_{2}\right)$ (resp. $z\left(\lambda_{3}\right)$ ) is attractive (resp.repulsive) for $f_{\lambda_{2}}^{n_{0}}$ (resp. $f_{\lambda_{3}}^{n_{0}}$ ). As the basin of $f_{\lambda_{2}}^{n_{0}}$ at $z\left(\lambda_{2}\right)$ contains a critical point, there exists $0 \leq i_{0} \leq n_{0}$ such that the sequence $d\left[z(\lambda), f_{\lambda}^{k n_{0}-i_{0}}\left(C_{f_{\lambda}}\right)\right]$ is converging to 0 around $\lambda_{2}$. Then, since $B^{\prime} \subset B \subset \mathcal{S}, d\left[z(\lambda), f_{\lambda}^{k n_{0}-i_{0}}\left(C_{f_{\lambda}}\right)\right]$ actually converges to 0 in $B^{\prime}$ which is impossible because $z\left(\lambda_{3}\right)$ is repulsive.

Remark 5.4 Two fundamental facts in Mane-Sad-Sullivan theory are the density of $\mathcal{R}$ in $X$ and the emerging concept of hyperbolic component: two elements lying in the same connected component of $\mathcal{R}$ are either both hyperbolic or both non-hyperbolic. This plays an important role in the approach of Fatou's conjecture on the density of hyperbolic rational maps. It turns out that these facts may be established by mean of elementary arguments similar to those used in the last steps of the proof of Theorem 5.2 .

We now aim to generalize Theorem 5.2 to the case of powers $\left(d d^{c} L\right)^{p}$. To this purpose we have to discuss the intersection of $p$ hypersurfaces $\operatorname{Per}_{1}\left(X, n, e^{2 i \pi \theta}\right)$. For any $N_{p}:=$ $\left(n_{1}, \ldots, n_{p}\right) \in\left(\mathbf{N}^{*}\right)^{p}$ and $\Theta_{p}:=\left(\theta_{1}, \ldots, \theta_{p}\right) \in E^{p}$ we define

$$
\operatorname{Per}\left(X, N_{p}, \Theta_{p}\right):=\operatorname{Per}\left(X, n_{1}, \theta_{1}\right) \cap \cdots \cap \operatorname{Per}\left(X, n_{p}, \theta_{p}\right) .
$$

As previously, the notation $\operatorname{Per}_{p}\left(X, N_{p}, \Theta_{p}\right)$ will refer to a co-dimension $p$ irreducible component of $\operatorname{Per}\left(X, N_{p}, \Theta_{p}\right)$. We then set:

$$
\mathcal{Z}_{p}(X, E):=\bigcup_{N_{p} \in\left(\mathbf{N}^{*}\right)^{p}, \Theta_{p} \in E^{p}} \operatorname{Per}_{p}\left(X, N_{p}, \Theta_{p}\right)
$$

Our generalization may be stated as follows.

Theorem 5.5 Let $E$ be a dense subset of $] 0,1\left[\right.$. Let $\left\{f_{\lambda}\right\}_{\lambda \in X}$ be an holomorphic family of rational maps of degree $d$ on $\mathbf{P}^{1}$. Then for any $1 \leq p \leq \operatorname{dim}_{\mathbf{C}} X$ :

$$
\operatorname{Supp}\left(d d^{c} L\right)^{p} \subset \overline{\mathcal{Z}}_{p}(X, E)
$$

Proof. We proceed by induction on $p$. Let us call $(\mathcal{H})_{p}$ the following assertion:

$(\mathcal{H})_{p}$ : For any complex manifold $X$ of dimension $n \geq p$ and any holomorphic family $\left\{f_{\lambda}\right\}_{\lambda \in X}$ parametrized by $X$ we have $\operatorname{Supp}\left(d d^{c} L\right)^{p} \subset \overline{\mathcal{Z}}_{p}(X, E)$.

According to Theorem $\left[5.2(\mathcal{H})_{1}\right.$ is true. Let us show that $(\mathcal{H})_{p}$ implies $(\mathcal{H})_{p+1}$. To this end we shall combine the following fact with $(\mathcal{H})_{1}$. 
Fact: Assume that $(\mathcal{H})_{p}$ is true. Let $U$ be an open set in $\mathbf{C}^{n}(n>p)$ and $\left\{f_{\lambda}\right\}_{\lambda \in U}$ be an holomorphic faamily. If $L\left(f_{\lambda}\right)$ is pluriharmonic on every $\operatorname{Per}_{p}\left(U, N_{p}, \Theta_{p}\right)$ then $\left(d d^{c} L\right)^{p+1} \equiv 0$ on $U$.

This fact, which is actually at the heart of our proof, will be established later. It is usefull to remark that a continuous function on an analytic set $Y$ is p.s.h. if and only if it is p.s.h. on the set of regular points of $Y$ (see [6], Theorem 1.7). We also recall that $L$ is continuous (see Corollary [3.4).

Let us consider an holomorphic family $\left\{f_{\lambda}\right\}_{\lambda \in X}$ and $\lambda_{0} \in S u p p\left(d d^{c} L\right)^{p+1}$. Pick an arbitrary small open set $U$ such that $\lambda_{0} \in U$. We have to show that $\mathcal{Z}_{p+1}(X, E) \cap U \neq \emptyset$. We may identify $U$ with an open set of $\mathbf{C}^{n}$. According to the above fact, there exist $N_{p} \in\left(\mathbf{N}^{*}\right)^{p}$ and $\Theta_{p} \in E^{p}$ such that $L$ is not pluriharmonic on $\operatorname{Per}_{p}\left(U, N_{p}, e^{2 i \pi \Theta_{p}}\right)$. This implies the existence of some regular curve $\Gamma$ contained in $\operatorname{Per}_{p}\left(U, N_{p}, e^{2 i \pi \Theta_{p}}\right)$ such that $d d^{c}\left(L_{\mid \Gamma}\right)$ does not vanish. Thus, Theorem 5.2 applied to the family $\left\{f_{\lambda}\right\}_{\lambda \in \Gamma}$ guarantees the existence of some $\operatorname{Per}_{1}\left(\Gamma, n_{p+1}, e^{2 i \pi \theta_{p+1}}\right)$. Then, setting $N_{p+1}:=\left(N_{p}, n_{p+1}\right)$ and $\Theta_{p+1}:=$ $\left(\Theta_{p}, \theta_{p+1}\right)$ we clearly may find some $\operatorname{Per}_{p+1}\left(U, N_{p+1}, e^{2 i \pi \Theta_{p+1}}\right)$ in $\operatorname{Per}_{p}\left(U, N_{p}, e^{2 i \pi \Theta_{p}}\right) \cap$ $\operatorname{Per}_{1}\left(U, n_{p+1}, e^{2 i \pi \theta_{p+1}}\right)$. Thus $\operatorname{Per}_{p+1}\left(U, N_{p+1}, e^{2 i \pi \Theta_{p+1}}\right) \subset \mathcal{Z}_{p+1}(X, E) \cap U$ and we are done.

Proof of the fact: By an elementary slicing argument, the positive current $\left(d d^{c} L\right)^{p+1}$ is identically vanishing on $U$ as soon as the positive measures obtained by restriction on the $(p+1)$-dimensional affine subspaces are vanishing. Let $S$ be the intersection of $U$ with such an affine subspace of $\mathbf{C}^{n}$ and set $L_{0}:=L_{\mid S}, \mu_{0}:=\left(d d^{c} L_{0}\right)^{p+1}$. We have to show that for every euclidean, $(p+1)$-dimensional, open ball $B \subset \subset S$, the measure $\mu_{0}$ vanishes on $\frac{1}{2} B$.

To this end, we introduce the solution $\tilde{L}_{0}$ of the Dirichlet-Monge- Ampère problem with data $L_{0}$ on $b B$. The function $\tilde{L}_{0}$ is continuous on $\bar{B}$, concides with $L_{0}$ on $b B$ and is p.s.h. maximal on $B$ (see [1]). By maximality, $\tilde{L}_{0} \geq L_{0}$ on $\bar{B}$. We also consider the set $\Sigma_{\varepsilon} \subset \frac{1}{2} B$ where $L_{0}$ and $\tilde{L}_{0}$ are $\varepsilon$-close:

$$
\Sigma_{\varepsilon}:=\left\{0 \leq \tilde{L}_{0}-L_{0} \leq \varepsilon\right\} \cap \frac{1}{2} B
$$

A theorem of Briend-Duval (see [3] or [26] Theorem A.10.2) states that

$$
\mu_{0}\left(\Sigma_{\varepsilon}\right) \leq C \varepsilon
$$

where the constant $C$ depends only on $L_{0}$ and $B$. It thus suffices to show that $\operatorname{Supp}\left(\mu_{0}\right) \cap$ $\frac{1}{2} B \subset \Sigma_{\varepsilon}$ for any $\varepsilon>0$.

The set $\mathcal{Z}_{p}(S, E)$ is an union of complex curves in $S$. Let $A$ be one of these curves, then $A$ is of the form $\operatorname{Per}_{p}\left(S, N_{p}, e^{2 i \pi \Theta_{p}}\right)$ and is therefore contained in $S \cap \operatorname{Per}_{p}\left(U, N_{p}, e^{2 i \pi \Theta_{p}}\right)$ (remark that it is the opposite inclusion which can be false), thus the function $L=L_{0}$ is, by assumption, harmonic on $A \cap B$. The function $\tilde{L}_{0}-L_{0}$ is thus subharmonic on $A \cap B$ and, by the maximum principle, identically vanishes. Therefore $\tilde{L}_{0}-L_{0}$ vanishes on $\mathcal{Z}_{p}(S, E) \cap B$.

Of course $\operatorname{Supp}\left(\mu_{0}\right) \subset \operatorname{Supp}\left(d d^{c} L_{0}\right)^{p}$ and, as $\left(\mathcal{H}_{p}\right)$ is supposed be true, $\operatorname{Supp}\left(d d^{c} L_{0}\right)^{p} \subset \overline{\mathcal{Z}}_{p}(S, E)$. Thus $\tilde{L}_{0}-L_{0}$ vanishes on $\operatorname{Supp}\left(\mu_{0}\right) \cap \frac{1}{2} B$, which is, therefore, contained in $\Sigma_{\varepsilon}$. 


\section{The measure of bifurcation}

The results of this section are mainly devoted two bifurcation theory in 1-dimension; in particular we define the bifurcation measure $\mu$ on the moduli space $\mathcal{M}_{d}$ of rational maps $\mathbf{P}^{1} \rightarrow \mathbf{P}^{1}$ and we establish some elementary facts about it. Neverthless the fact that the bifurcation currents $\left(d d^{c} L\right)^{p}$ have finite mass on $\mathcal{H}_{d}$ will be established in any dimension, that is in $\mathcal{H}_{d}\left(\mathbf{P}^{k}\right)$.

The group $P S L(2, \mathbf{C})$ of Möbius transformation acts on the space $\mathcal{H}_{d}\left(\mathbf{P}^{1}\right)$ by conjugation. Two conjugated rational function $f_{1}, f_{2} \in \mathcal{H}_{d}\left(\mathbf{P}^{1}\right)$ have the same dynamics, therefore in order to study the stability of holomorphic families of rational functions one considers, instead of $\mathcal{H}_{d}\left(\mathbf{P}^{1}\right)$, the moduli space $\mathcal{M}_{d}:=\mathcal{H}_{d}\left(\mathbf{P}^{1}\right) / P S L(2, \mathbf{C})$.

Remark 6.1 The moduli space $\mathcal{M}_{d}$ is a normal, quasi-projective variety (see [27, Remark p.43); the proof requires some effort because $P S L(2, \mathbf{C})$ is not compact and its action on $\mathcal{H}_{d}\left(\mathbf{P}^{1}\right)$ is not free (indeed for some $f \in \mathcal{H}_{d}\left(\mathbf{P}^{1}\right)$ the isotropy group $A u t(f):=\{\varphi \in$ $\left.P S L(2, \mathbf{C}) ; \varphi^{-1} \circ f \circ \varphi=f\right\}$ is not trivial). Here we recall some usefull facts about $\mathcal{M}_{d}$ :

(i) the canonical projection $\Pi: \mathcal{H}_{d}\left(\mathbf{P}^{1}\right) \rightarrow \mathcal{M}_{d}$ is open;

(ii) for all $f \in \mathcal{H}_{d}\left(\mathbf{P}^{1}\right)$, the isotropy group Aut $(f)$ is finite and locally there is a complex submanifold $V$, invariant by the action of Aut $(f)$, transvers at $f$ to the orbit of $f$, such that $\Pi(V)$ is open in $\mathcal{M}_{d}$ and the canonical projection $\Pi$ induces a biholomorphism $V /$ Aut $(f) \rightarrow \Pi(V)$;

(iii) the set of all $f \in \mathcal{H}_{d}\left(\mathbf{P}^{1}\right)$ such that $A u t(f) \neq\left\{i d_{\mathbf{P}^{1}}\right\}$ is an analytic subset $Z$ of $\mathcal{H}_{d}\left(\mathbf{P}^{1}\right)$ and $\operatorname{sing}\left(\mathcal{M}_{d}\right) \subset \Pi(Z)$.

(In general $\operatorname{sing}\left(\mathcal{M}_{d}\right) \neq \Pi(Z)$, e.g. $\mathcal{M}_{2}=\mathbf{C}^{2}$ is smooth, and $\Pi(Z)$ is a cubic curve of $\mathbf{C}^{2}=\mathcal{M}_{2}$ (see [19], Corollary 5.3). But, for $d>2, \mathcal{M}_{d}$ has singular points). It follows that if $f \notin Z$, then $P S L(2 ; \mathbf{C}) \times V \simeq \Pi^{-1}(\Pi(V))$, therefore

(iv) $\mathcal{H}_{d}\left(\mathbf{P}^{1}\right) \backslash Z \rightarrow \mathcal{M}_{d} \backslash \Pi(Z)$ is a principal bundle

Since $\operatorname{dim}_{\mathbf{C}} P S L(2, \mathbf{C})=3$ and all isotropy groups $A u t(f)$ are finite, hence each orbit is a complex 3 -fold and $\operatorname{dim}_{\mathbf{C}} \mathcal{M}_{d}=\operatorname{dim}_{\mathbf{C}} \mathcal{H}_{d}\left(\mathbf{P}^{1}\right)-3=2(d-1)$. The Lyapunouv exponent $L(f), f \in \mathcal{H}_{d}\left(\mathbf{P}^{1}\right)$, which is invariant for the action of $\operatorname{PSL}(2, \mathbf{C})$, is constant on the orbits and, if $p>2(d-1)$, the current $\left(d d^{c} L\right)^{p}$ vanish identically in $\mathcal{H}_{d}\left(\mathbf{P}^{1}\right)$. Therefore in order to define a measure taking a sufficiently high power of $d d^{c} L$ it is necessary to consider the function $\hat{L}: \mathcal{M}_{d} \rightarrow \mathbf{R}$ induced from $\mathcal{H}_{d}\left(\mathbf{P}^{1}\right) \stackrel{L}{\longrightarrow} \mathbf{R}$.

Proposition 6.2 The function $\hat{L}$ is continous, bounded from below and p.s.h. on $\mathcal{M}_{d}$.

Proof. By [23, $L$ is bounded from below. Using Corollary 3.4] it is enough to remark that, by means of Remark 6.1 (iv), $\hat{L}$ is p.s.h. on $\mathcal{M}_{d} \backslash \Pi(Z)$ and thus (see [6], Thm. 1.7) on the whole $\mathcal{M}_{d}$.

Now the currents $\left(d d^{c} \hat{L}\right)^{p}, 1 \leq p \leq 2(d-1)$, are well defined on $\mathcal{M}_{d}$. In particular, we shall call measure of bifurcartion the measure $\mu:=\left(d d^{c} \hat{L}\right)^{2(d-1)}$. 
Proposition 6.3 The measure of bifurcation $\mu$ does not vanish identically.

Proof. For $d \geq 2$ fixed, let $f_{0} \in \mathcal{H}_{d}\left(\mathbf{P}^{1}\right)$ be a non-flexible Lattès map (e.g. $f_{0}$ is the map associated to an immaginary quadratic number field, see [21] Lemma 5.4), then all the Lattès map which belong to a small neighbourhood of $f_{0}$ in $\mathcal{H}_{d}\left(\mathbf{P}^{1}\right)$ are coniugated to $f_{0}$. Let $V$ be a complex submanifold in a neighbourhood of $f_{0}$ as in Remark 6.1 (ii); since the function $L(f)$ takes its minimum value $\log \sqrt{d}$ exactly when $f$ is a Lattès map, hence $f_{0}$ is a point of strict minimum for $L_{\mid V}$. As $\operatorname{dim}_{\mathbf{C}} V=2(d-1)$ we shall see that $f_{0} \in \operatorname{Supp}\left(d d^{c} L_{\mid V}\right)^{2(d-1)}$, i.e. $\Pi\left(f_{0}\right) \in \operatorname{Supp}(\mu)$. For every small, euclidean, open ball $B \subset V$ centered at $f_{0}$ there is a suitable constant $c$ such that $L\left(f_{0}\right)<c<L(f)$, for every $f \in B$; so the function $L_{\mid V}-c$ does not take its minimum on $\bar{B}$ at the boundary, therefore (see Theorem A in [1]) it is not maximal, that is $\left(d d^{c} L_{\mid V}\right)^{2(d-1)}$ does not vanish identically on $B$.

In order to see that $\mu$ has finite mass (see Proposition 6.6) we shall show that $L$ extends from $\mathcal{H}_{d}$ to the whole projective space across the hypersurface $\Sigma_{d}$ and that the powers of $d d^{c} L$ have finite mass on $\mathcal{H}_{d}$. Since these results hold for holomorphic maps $\mathbf{P}^{k} \rightarrow \mathbf{P}^{k}$, $k \geq 1$, we believe that it is usefull to present them in this more general case.

First of all let us recall that $\mathcal{H}_{d}\left(\mathbf{C}^{k+1}\right)=\mathbf{C}^{N} \backslash \tilde{\Sigma}_{d}$, see Subsection 1.1 and that from (4.1) and Proposition 4.9 it follows:

$$
L(F)=H(F)-\frac{k+1}{d^{k}} \log |\operatorname{Res}(F)|+c s t .
$$

for every polynomial map $F \in \mathcal{H}_{d}\left(\mathbf{C}^{k+1}\right) \subset \mathbf{C}^{N+1}$.

Proposition 6.4 The function $H$ extends from $\mathcal{H}_{d}\left(\mathbf{C}^{k+1}\right)$ to a p.s.h. function on the whole $\mathbf{C}^{N+1}$ and the function $L(f)$ extends from $\mathcal{H}_{d}\left(\mathbf{P}^{k}\right)$ to a function $L_{\text {loc }}^{1}\left(\mathbf{P}^{N}\right)$. Moreover there is a $(1,1)$-current $R$, positive and closed on $\mathbf{P}^{N}$ such that $\pi^{*} R=d d^{c} H$ and

$$
d d^{c} L=R-\frac{k+1}{d^{k}}\left[\Sigma_{d}\right] .
$$

Proof. Let $K \subset \mathbf{C}^{N+1}$ be a compact, let us check that $H$ is bounded from above on $\overline{K \cap \mathcal{H}_{d}}\left(\mathbf{C}^{k}\right)$. By definition (see (4.2) ):

$$
H(F)=\int_{C_{f}} g_{F} \sum_{j=0}^{k-1} T_{f}^{j} \wedge \omega^{k-j-1}+\int_{\mathbf{P}^{k}} \log \left\|J_{F}\right\|_{0} \omega^{k} .
$$

Then, as $g_{F}$ is locally bounded from above (see Remark 1.3), one concludes taking into account the following formulas: $\int_{C_{f}} \sum_{j=0}^{k-1} T_{f}^{j} \wedge \omega^{k-j-1}=k \operatorname{deg}\left(C_{f}\right)$ and $\int_{\mathbf{P}^{k}} \log \left\|\tilde{J}_{F}\right\|_{0} \omega^{k}=$ $=\int_{\mathbf{C}^{k+1}} \log \left|\operatorname{det}\left(F^{\prime}(Z)\right)\right| m$ (Lemma 1.4). From this, since $H$ is p.s.h. on $\mathcal{H}_{d}\left(\mathbf{C}^{k+1}\right.$ ) (see the Claim in the proof of Theorem 4.5), it follows that $H$ extends to a p.s.h. function on the whole $\mathbf{C}^{N+1}$. Thus the right hand side of (6.1) belongs to $L_{l o c}^{1}\left(\mathbf{C}^{N+1}\right)$ and extends $L(F)$ as a 0 -homogeneous $L_{l o c}^{1}$ function on $\mathbf{C}^{N+1}$. Thus $L(f)$ is well defined in the whole $\mathbf{P}^{N}$. Choosing an holomorphic section $U \stackrel{\sigma}{\longrightarrow} \mathbf{C}^{N+1} \backslash\{0\}$ on an open subset $U$ di $\mathbf{P}^{N}$, we get $\forall f \in U$,

$$
L(f)=H(\sigma \circ f)-\frac{k+1}{d^{k}} \log |\operatorname{Res}(\sigma \circ f)|+c s t .
$$


As $d d^{c}(H \circ \sigma)$ does not depend on $\sigma$, one defines a positive, closed current $R$ on $\mathbf{P}^{N}$ such that $\pi^{*} R=d d^{c} H$. Then from (6.3) it follows (6.2).

The sum $L(f)$ of Lyapunouv exponents is bounded from below (see Theorem 1 in [3]), thus, as in the 1-dimensional case, the powers of $d d^{c}\left(L_{\mid \mathcal{H}_{d}\left(\mathbf{P}^{N}\right)}\right)$ are well defined; but to show that these currents have finite mass takes some work.

Proposition 6.5 For $1 \leq p \leq N$,

$$
\int_{\mathcal{H}_{d}\left(\mathbf{P}^{k}\right)}\left(d d^{c} L\right)^{p} \wedge \omega^{N-p}<\infty
$$

and the trivial extension $S_{(p)}$ of $\left(d d^{c}\left(L_{\mid \mathcal{H}_{d}}\right)\right)^{p}$ to the whole $\mathbf{P}^{N}$ is well defined.

Remark. We recall that, by definition of trivial extension, $S_{(p)}$ is the positive, closed, $(p, p)$-current on $\mathbf{P}^{N}$ characterized by

(i) $S_{(p)}=\left(d d^{c} L\right)^{p}$ on $\mathcal{H}_{d}\left(\mathbf{P}^{k}\right)$

(ii) $\chi_{\Sigma_{d}} \cdot S_{(p)}=0$, where $\chi_{\Sigma_{d}}$ is the caracteristic function.

Proof. From (6.2) it follows $d d^{c} L \leq R$, thus $d d^{c} L$ has finite mass on $\mathcal{H}_{d}\left(\mathbf{P}^{k}\right)$ and its trivial extension to $\mathbf{P}^{N}$ is the positive, closed current $S_{(1)}:=\left(1-\chi_{\Sigma_{d}}\right) R$.

Now we shall argue by induction, assuming that the trivial extension $S_{(p)}$ of $\left(d d^{c}\left(L_{\mid \mathcal{H}_{d}}\right)\right)^{p}$ to the whole $\mathbf{P}^{N}$ is well defined (and, of course, positive and closed). There is a smooth, closed (1,1)-form $\alpha$ on $\mathbf{P}^{N}$ such that $S_{(1)}-\alpha=d d^{c} u$ and, by means of the regularization theorem of Demailly $([7])$, there are a sequence $\left\{u_{n}\right\}$ of smooth functions decreasing to $u$ and a sequence $\left\{\lambda_{n}\right\}$ of continuous functions decreasing to $\nu\left(S_{(1)},.\right)$, such that $S_{n}:=\alpha+d d^{c} u_{n} \rightarrow S_{(1)}$ and $S_{n}+\lambda_{n} \omega \geq 0$.

We can estimate the mass of $S_{(p)} \wedge\left(S_{n}+\lambda_{n} \omega\right)$ (which is a positive current) as follows:

$$
\begin{gathered}
\left\|S_{(p)} \wedge\left(S_{n}+\lambda_{n} \omega\right)\right\|=\int_{\mathbf{P}^{N}} S_{(p)} \wedge \alpha \wedge \omega^{N-p-1}+ \\
+\int_{\mathbf{P}^{N}} S_{(p)} \wedge d d^{c} u_{n} \wedge \omega^{N-p-1}+\int_{\mathbf{P}^{N}} \lambda_{n} S_{(p)} \wedge \omega^{N-p}
\end{gathered}
$$

Let us look at the right hand side: the first term is constant, the second vanishes. Since $L$ (and therefore $H$ ) is bounded from below on $\mathbf{P}^{N} \backslash \Sigma_{d}=\mathcal{H}_{d}\left(\mathbf{P}^{k}\right)$, hence Lelong numbers of $S_{(1)}$ vanish outside $\Sigma_{d}$. Thus $\lambda_{n}$ decreases to $\nu\left(S_{(1)},.\right) \chi_{\Sigma_{d}}$, but $\chi_{\Sigma_{d}} S_{(p)}=0$, therefore $\lambda_{n} S_{(p)} \rightarrow 0$.

This means that $S_{(p)} \wedge\left(S_{n}+\lambda_{n} \omega\right)$ is bounded in mass, thus we can assume that it converges to a positive current $Q$. But $S_{(p)} \wedge \lambda_{n} \omega \rightarrow 0$, thus $Q$ is closed. Therefore we can set $S_{(p+1)}:=\left(1-\chi_{\Sigma_{d}}\right) Q$.

Coming back to 1-dimensional case, we can establish:

Proposition 6.6 The bifurcation measure $\mu$ has finite mass on $\mathcal{M}_{d}$. 
Proof. Since $\hat{L}$ is bounded from below, the measure $\mu$ does not charge analytic subsets thus from Remark 6.1 (iv) and the previous Proposition we get:

$$
\begin{gathered}
\int_{\mathcal{M}_{d}} \mu=\int_{\mathcal{M}_{d} \backslash \Pi(Z)}\left(d d^{c} \hat{L}\right)^{2(d-1)}= \\
=\int_{\mathcal{H}_{d}\left(\mathbf{P}^{1}\right) \backslash Z}\left(d d^{c} L\right)^{2(d-1)} \wedge \omega^{3}=\int_{\mathcal{H}_{d}\left(\mathbf{P}^{1}\right)}\left(d d^{c} L\right)^{2(d-1)} \wedge \omega^{3}<\infty .
\end{gathered}
$$

Remark 6.7 Since the hypersurfaces $\operatorname{Per}\left(\mathcal{H}_{d}\left(\mathbf{P}^{1}\right), n, e^{2 i \pi \theta}\right)$ are invariant for the action of $P S L(2, \mathbf{C})$ there are no difficulties in order to get, from Theorems 5.2 and 5.5 , the corresponding statement for $\hat{L}$. Infact the following hold: $\operatorname{Supp}\left(d d^{c} \hat{L}\right)=\overline{\mathcal{Z}}_{1}\left(\mathcal{M}_{d}\right)$ and for $1<p \leq 2(d-1), \operatorname{Supp}\left(d d^{c} \hat{L}\right)^{p} \subset \overline{\mathcal{Z}}_{p}\left(\mathcal{M}_{d}\right)$. In particular $\operatorname{Supp}(\mu) \subset \overline{\mathcal{Z}}_{2(d-1)}\left(\mathcal{M}_{d}\right)$

We say that a point $x \in \mathcal{M}_{d}$ is chaotic if the Julia set of any $f \in \Pi^{-1}(x)$ is $\mathbf{P}^{1}$.

Proposition 6.8 In any neighbourhood of a point of Supp $(\mu)$ there are uncountably many chaotic points.

$\underline{\text { Proof }}^{1}$. Consider $\left\{\theta \in \mathbf{R} / \lim \sup _{n \rightarrow \infty} \frac{\log \log \left(1 /\left|\theta^{n}-1\right|\right)}{n}<\log d\right\}$ and use this open dense and uncountable subset of $\mathbf{R}$ to define $E$ and $\mathcal{Z}_{p}\left(\mathcal{H}_{d}\left(\mathbf{P}^{1}\right), E\right.$ ) (see [20]). If $z_{0}$ is a periodic points of $f \in \mathcal{H}_{d}\left(\mathbf{P}^{1}\right)$ with multiplier $e^{2 i \pi \theta}, \theta \in E$, then $z_{0}$ is a Cremer point; therefore any $f \in \mathcal{Z}_{2(d-1)}\left(\mathcal{H}_{d}\left(\mathbf{P}^{1}\right)\right)$ has $2(d-1)$ Cremer points and (see [25], Corollary 2) is chaotic.

\section{Examples and applications.}

\subsection{Geodesics on the space of the Kähler metrics.}

Let $M$ be a compact Kähler manifold of dimension $k$ with a fixed Kähler metric $\omega$. In order to discuss extremal (e.g. Einstein and of constant scalar curvature) Kähler metrics it is usefull to consider the space $H_{\omega}$ of Kähler metrics with the same Kähler class of $\omega$ (see e.g. [5]). It can be viewed also as the space of Kähler potentials, that is

$$
H_{\omega}:=\left\{\phi \in \mathcal{C}^{\infty}(M) ; \omega+i \partial \bar{\partial} \phi>0\right\} / \sim
$$

where $\phi_{1} \sim \phi_{2}$ if and only if $\phi_{1}-\phi_{2}$ is a constant. Endowing $H_{\omega}$ with a suitable metric it turns out that $H_{\omega}$, as Riemannian manifold, is an infinite dimensional symmetric space and there is a (unique) Levi-Civita connection whose curvature is covariant constant (see [24] and [5]).

For such a connection the equation of geodesic is

$$
(i \partial \bar{\partial} \phi+\omega)^{k+1}=0
$$

\footnotetext{
${ }^{1}$ We would like to thank T.C. Dinh who told us the possibiliy to use Shishikura's theorem here.
} 
This means that $\phi$ is a smooth real function defined on $[0,1] \times M$ (in this case one understands the $\bar{\partial}$ operator as the one on the cylinder $[0,1] \times S^{1}$ with its natural complex structure) or, for complex geodesic, it is more generally defined on $X \times M$ where $X$ is a Riemann surface. It must be point out that very few explicit examples of these geodesics are known, thus the following remark may have some interest.

Let $M=\mathbf{P}^{k}$ and $\omega$ be the Fubini-Study metric, Corollary 4.6 says that any holomorphic family $\left\{f_{\lambda}\right\}_{\lambda \in X}$ of endomorphisms of $\mathbf{P}^{k}$ defines a "geodesic" $\phi:=g_{F_{\lambda}}$. Of course the behaviour of $d d^{c} g_{F_{\lambda}}+\omega$ is very far from the desidered regularity, but there is at least one case in which holomorphic dynamics may give interesting examples: let $M=\mathbf{P}^{1}$ and $\left\{f_{\lambda}\right\}_{\lambda \in X}$ be a family of flexible Lattès maps (see [21], Ch. 8.3), then the functions $g_{F_{\lambda}}: \mathbf{P}^{1} \rightarrow \mathbf{R}$ are smooth outside a finite set.

\subsection{Attractor in $\mathrm{P}^{2}$}

Definition 7.1 Let $\left\{f_{\lambda}\right\}_{\lambda \in X}$ be a one parameter holomorphic family (i.e. $X$ is an open subset of $\mathbf{C}$ ) of endomorphisms of $\mathbf{P}^{k}$ and $Y$ be a complex subspace of $X \times \mathbf{P}^{k}$ of pure dimension $q$. We shall say that the Green function $G_{\lambda}$ is maximal on $Y$ if and only if, for every holomorphic section $\mathbf{P}^{k} \supset U \stackrel{\sigma}{\longrightarrow} \mathbf{C}^{k+1} \backslash\{0\}$,

$$
\left(d d^{c}\left(G_{\lambda} \circ \sigma\right)\right)^{q}=0 \text { on } Y \cap(X \times U) .
$$

Although the Green function depends on the choice of the lift of $f_{\lambda}$, the definition is well posed since $d d^{c}\left(G_{\lambda} \circ \sigma\right)$ does not depend on the particular family of lifts $\left\{F_{\lambda}\right\}_{\lambda \in W}$ chosen in an open subset $W$ of $X$.

With this definition we can give the following formulation of Corollary 4.6

Proposition 7.2 Let $\left\{f_{\lambda}\right\}_{\lambda \in X}$ be a one parameter holomorphic family of endomorphisms of $\mathbf{P}^{k}$. Then $G_{\lambda}$ is maximal on $X \times \mathbf{P}^{k}$. Moreover $G_{\lambda}$ is maximal on $C_{X}$ if and only if $L\left(f_{\lambda}\right)$ is harmonic.

Now we shall apply this Proposition to a particular case. For $\varepsilon \in \mathbf{C}$, consider the rational $\operatorname{map} \mathbf{P}^{2} \rightarrow \mathbf{P}^{2}$ defined by

$$
f_{\varepsilon}=\left[P(z, w): Q(z, w): t^{d}+\varepsilon R(z, w)\right]
$$

where $P, Q, R$ are homogeneous polynomials of degree $d \geq 2$ such that $(P, Q)$ is non degenerate and the induced rational function

$$
f=[P(z, w): Q(z, w)]
$$

is strictly critically finite. It is usefull to consider the line $\mathcal{R}_{\infty}:=\{t=0\}$ as the line at infinity of the complex plane $\mathbf{C}^{2} \simeq\left\{[z: w: 1] \in \mathbf{P}^{2}\right\}$. If $a \in \mathcal{R}_{\infty}$, we shall denote by $\mathcal{R}_{a}$ the line passing through the origin $[0: 0: 1]$ and $a$. The map $f_{\varepsilon}$ preserves lines through the origin and moves them in a chaotic way, since $f$ is chaotic, infact identifying $\mathcal{R}_{\infty}$ with $\mathbf{P}^{1}$ we get $f_{\varepsilon}\left(\mathcal{R}_{a}\right) \cap \mathcal{R}_{\infty}=\{f(a)\}$.

For $|\varepsilon|<<1$ the only Fatou components is the superattractive basin of the origin (see 12] Lemma 2.1). Moreover for $|\varepsilon|<<1$ the map $f_{\varepsilon}$ has an attractor $A$ contained in a neighbourhood of the line at infinity, which intersect any line passing trhough origin (ibidem, Lemma 2.2).

Our aim is to show that, for $|\varepsilon|<<1$, the family $\left\{f_{\varepsilon}\right\}$ is stable in the following sense: 
Proposition 7.3 The function $\varepsilon \rightarrow L\left(f_{\varepsilon}\right)$ is harmonic in a neighbourhood of $0 \in \mathbf{C}$.

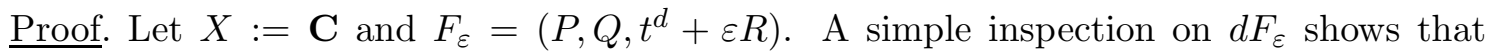
$C_{f_{\varepsilon}}=\mathcal{R}_{\infty} \cup\left(\cup_{c \in C_{f}} \mathcal{R}_{c}\right)$, thus the critical set does not depend on $\varepsilon$ and

$$
C_{X}=\left(X \times \mathcal{R}_{\infty}\right) \cup\left(\cup_{c \in C_{f}} X \times \mathcal{R}_{c}\right) .
$$

Let $c \in C_{f}$; then by hypothesis, there exists $j, k \in \mathbf{N}^{*}$ such that, $a:=f^{j}(c)$ and $f^{k}(a)=a$. This means that $\mathcal{R}_{a}$ is fixed by $f_{\varepsilon}^{k}$. Thus we can consider the family $f_{\varepsilon \mid \mathcal{R}_{a}}^{k}$ as a family of endomorphisms of $\mathcal{R}_{a}$. From Proposition $[7.2$ it follows that, for this family, the Green function is maximal on $X \times \mathcal{R}_{a}$. But all the power of $F_{\varepsilon}$ have the same Green function $G_{F_{\varepsilon}}$. That is, for every section $U \stackrel{\sigma}{\longrightarrow} \mathbf{C}^{3} \backslash\{0\}$, the function $G_{F_{\varepsilon}} \circ \sigma$ is maximal on $X \times\left(U \cap \mathcal{R}_{a}\right)$. Since $f_{\varepsilon}^{j}\left(\mathcal{R}_{c}\right)=\mathcal{R}_{a}$ and $G_{F_{\varepsilon}} \circ \sigma \circ f_{\varepsilon}^{j}=G_{F_{\varepsilon}}\left(h F_{\varepsilon}^{j} \circ \sigma^{\prime}\right)=d^{j} G_{F_{\varepsilon}} \circ \sigma^{\prime}+\log \left|h \circ \sigma^{\prime}\right|$, for a suitable section $\sigma^{\prime}$ and an holomorphic, never vanishing, function $h$, we get that $G_{F_{\varepsilon}}$ is maximal on $X \times \mathcal{R}_{c}$.

Now we shall show that choosing $V=\{|\varepsilon|<<1\}$, the function $G_{F_{\varepsilon}}$ is maximal on $V \times \mathcal{R}_{\infty}$. Let $u \in \mathcal{R}_{\infty} \simeq \mathbf{P}^{1}$ be a periodic point for $f$ (that is $f_{\varepsilon}^{j}\left(\mathcal{R}_{u}\right)=\mathcal{R}_{u}$ for some $j)$. From the proof of Lemma 2.2 in [12] it follows that there is an open neighbourhood $W$ of $\mathcal{R}_{\infty}$ in $\mathbf{P}^{2}$ such that, if $|\varepsilon|<<1$, then $f_{\varepsilon}(W) \subset \subset W$; thus the family $\left\{f_{\varepsilon}^{n}(u)\right\}_{n \in \mathbf{N}}$ is a normal in $V$ (as functions of $\varepsilon$ ). Therefore $\varepsilon \rightarrow G_{F_{\varepsilon}}(\sigma(u))$ is harmonic on $V$. That's enough since these points $u$ are dense in $\mathcal{R}_{\infty}$.

\subsection{The measure of bifurcation on $\mathcal{M}_{2}$}

As we have already recalled the moduli space $\mathcal{M}_{2}$ of the rational functions of degree 2 can be identified biholomorphically and in a canonical way with $\mathbf{C}^{2}$ (see [19]). Such an identification involves the affine structure since, for every $\eta \in \mathbf{C}, \operatorname{Per}\left(\mathcal{M}_{2}, 1, \eta\right)$ is a straight line of $\mathbf{C}^{2}$. In particular the Mandelbrot family $\left\{z^{2}+c ; c \in \mathbf{C}\right\}$ coincides with the straigh line $\operatorname{Per}\left(\mathcal{M}_{2}, 1,0\right)$ of rational functions with a superattractive fixed point.

Proposition 7.4 The Mandelbrot family is disjoint from the support of the bifurcation measure $\mu$ on $\mathcal{M}_{2}$.

Proof. Since every $f_{0} \in \operatorname{Per}\left(\mathcal{M}_{2}, 1,0\right)$ has a superattractive fixed point, there is an open subset $V, \operatorname{Per}\left(\mathcal{M}_{2}, 1,0\right) \subset V \subset \mathcal{M}_{2}$, such that every $f \in V$ has an attracting fixed point.

For every $f \in \mathcal{M}_{2}$ the number of attracting or indifferent cycles is $\leq 2$ (see [25], Corollary 1), therefore $V \cap \mathcal{Z}_{2}\left(\mathcal{M}_{2}, E\right)=\emptyset$. Thus from Theorem 5.5 it follows that $V$ is disjoint from $\operatorname{Supp}\left(d d^{c} \hat{L}\right)^{2}=\operatorname{Supp}(\mu)$.

Remark 7.5 Let us point out that it is not necessary to use Shishikura's result. First we can assume that if $f \in V$, then the attractive fixed point depends holomorphically on $f$. Consider an holomorphic disc $\left\{f_{\lambda}\right\}_{\lambda \in \Delta}$ contained in $V \cap \operatorname{Per}_{1}\left(\mathcal{M}_{2}, n, \theta\right)$. If $\hat{L}$ is not harmonic on this disc, then from Theorem 5.2 there is an holomorphic function $z(\lambda)$ on an open disc $\Delta^{\prime} \subset \Delta$ such that $f_{\lambda}^{n}(z(\lambda))=z(\lambda)$ and two values $\lambda_{1}, \lambda_{2} \in \Delta^{\prime}$ such that $z\left(\lambda_{1}\right)$ is repulsive and $z\left(\lambda_{2}\right)$ is attractive. Thus $f_{\lambda_{2}}$ has two attracting points, and it is stable in $\mathcal{M}_{2}$. This contradicts the fact that $f_{\lambda_{2}} \in \operatorname{Per}_{1}\left(\mathcal{M}_{2}, n, \theta\right) \subset \operatorname{Supp}\left(d d^{c} \hat{L}\right)$. Therefore $\hat{L}$ is pluriharmonic on every $\operatorname{Per}_{1}(V, n, \theta)$; from the Fact in the proof of Theorem 5.5 it follows that $\left(d d^{c} \hat{L}\right)^{2}=0$. 


\section{Appendix}

\section{Proof of proposition 4.3}

Let $u$ be a locally bounded function on $X$; using (4.7) with $R=u$, Fubini Theorem and a suitable partition of the unity it follows that, for a.e. $y \in Y, u_{y}=u_{\mid \pi^{-1}(y)}$. Since $T$ is positive and closed, then the slices of $T$ exist (see [28, (10.3)); from (4.7), since slicing commutes with the operators $d, \partial$ and $\bar{\partial}$ (see [28] (10.4)), it follows that, for a.e. $y \in Y$, $T_{y}$ is a current on $\pi^{-1}(y)$, positive and closed. Thus $u_{y} T_{y}$ is well defined. By definition $d d^{c} u_{h} \wedge T=d d^{c}\left(u_{h} \wedge T\right)$, thus we shall argue by recurrence and, in order to finish the proof, it is enough to show $u_{y} T_{y}=(u T)_{y}$.

This obviously holds if $u \in \mathcal{C}^{\infty}(X)$. Fix $\phi$ and $\psi$ as above and let $K$ be a compact such that $\operatorname{supp}(\psi) \subset K$. For a.e. $y \in Y$

$$
\left|\int_{\pi^{-1}(y)} u_{y} T_{y} \wedge \iota_{y}^{*} \psi\right| \leq \sup _{K}|u| C_{\psi}\left\|T_{y}\right\|_{K \cap \pi^{-1}(y)}
$$

therefore by means of Lebesgue Theorem

$$
\left|\int_{Y}\left(\int_{\pi^{-1}(y)} u_{y} T_{y} \wedge \iota_{y}^{*} \psi\right) \phi\right| \leq \sup _{K}|u| C_{\phi, \psi}\|T\|_{K}
$$

and the left hand side is well defined.

That's all, because this inequality shows that the operator $\Phi(u)=$ $=\int_{Y}\left(\int_{\pi^{-1}(y)} T_{y} \wedge \iota_{y}^{*}(u \psi)\right) \phi$ can be continously extended from $\mathcal{C}^{\infty}(X)$ to $L^{\infty}(X)$.

\section{Proof of Proposition 4.10}

In order to compute the constant $C_{d, k}$ it is enough to consider a particular $F$; infact if we take $F\left(z_{0}, \ldots, z_{k}\right):=\left(z_{0}^{d}, \ldots, z_{k}^{d}\right)$, then $\operatorname{Res}(F)=1$ by Proposition 1.1] and from Proposition 4.9, it follows

$$
C_{d, k}=B(F)
$$

Claim 1: $L(F)=(k+1) \log (d)$.

Proof of the Claim 1. A direct computation for this particular $F$ shows that $G_{F}(1, u)=$ $\log ^{+}\|u\|_{m}$, where $\|u\|_{m}=\max \left\{\left|u_{j}\right|, 1 \leq j \leq k\right\}$, for every $u \in \mathbf{C}^{k}$ and that if $(1, u)$ is a repulsive point for $F$, then $\left|u_{j}\right|=1$, for $1 \leq j \leq k$. Therefore (by the equidistribution of repulsive points with respect to $\left.\mu_{F}\right)$ we get $G_{F}(1, u)=0$ in $\operatorname{supp}\left(\mu_{f}\right)$. On the other hand $\operatorname{det} F^{\prime}(1, u)=d^{k+1} u_{1}^{d-1} \ldots u_{k}^{d-1}$ and, by definition, $\left\|J_{F}([1: u])\right\|_{G_{F}}=$ $e^{-D G_{F}(1, u)}\left|\operatorname{det} F^{\prime}(1, u)\right|$. Summing up we get that, in $\operatorname{Supp}\left(\mu_{f}\right)$,

$$
\log \left\|J_{F}([1: u])\right\|_{G_{F}}=(k+1) \log d+(d-1) \sum_{j=1}^{k} \log \left|u_{j}\right|=(k+1) \log d .
$$

The claim follows from this last equation and Lemma 1.4 (1).

Claim 2: $H(F)=(k+1)\left(C_{d, k-1}+\log d-(d-1) \frac{1}{2}\left(1+\frac{1}{2}+\cdots+\frac{1}{k}\right)\right)$ Proof of the Claim 2. The critical set $C_{F}$ is the union of the hyperplanes $H_{s}=\left\{z_{s}=0\right\}$, $0 \leq s \leq k$. By means of an obvious identification $H_{s} \simeq \mathbf{C}^{k}$ the restriction $\hat{F}$ of $F$ 
to each of these hyperplanes is of the form $\hat{F}\left(z_{0}, \ldots, z_{k-1}\right)=\left(z_{0}^{d}, \ldots, z_{k-1}^{d}\right)$. But again $B(\hat{F})=C_{d, k-1}$. Therefore from (4.3) it follows

$$
\int_{C_{F}} g_{F} \sum_{j=0}^{k-1} T_{F}^{j} \wedge \omega^{k-1-j}=\sum_{s=0}^{k} \int_{H_{s}} g_{F} \sum_{j=0}^{k-1} T_{F}^{j} \wedge \omega^{k-1-j}=(k+1) B(\hat{F})=(k+1) C_{d, k-1} .
$$

From Lemma $1.4(2)$

$$
\int_{\mathbf{P}^{k}} \log \left\|J_{F}\right\|_{0} \omega^{k}=\int_{\mathbf{C}^{k+1}} \log \left|\operatorname{det} F^{\prime}\right| m=(k+1) \log d+(d-1) \sum_{j=0}^{k} \int_{\mathbf{C}^{k+1}} \log \left|z_{j}\right| d m,
$$

and by means of an elementary calculation

$$
\int_{\mathbf{P}^{k}} \log \left\|J_{F}(z)\right\|_{0} \omega^{k}=(k+1) \log d-(k+1)(d-1) \frac{1}{2}\left(1+\frac{1}{2}+\cdots+\frac{1}{k}\right) .
$$

Putting (17.3)-(7.4) in (4.2) we get the Claim.

Now putting (7.2) and the two claims in (4.1) we get

$$
C_{d, k-1}-(d-1) C_{d, k}-(d-1) \frac{1}{2} \sum_{j=1}^{k} \frac{1}{j}=0 .
$$

Now to finish it is enough to calculate $C_{d, 1}$; for $k=1$ the map $F$ is given by $F\left(z_{0}, z_{1}\right)=$ $\left(z_{0}^{d}, z_{1}^{d}\right)$ and in this case (4.1) gives

$$
L(F)=g_{F}(0,1)+g_{F}(1,0)+\int_{\mathbf{P}^{1}} \log \left\|J_{F}(z)\right\|_{0} \omega-2(d-1) B(F) ;
$$

now by Claim $1, L(F)=2 \log d$, by (7.4), $\int_{\mathbf{P}^{1}} \log \left\|J_{F}(z)\right\|_{0} \omega=2 \log d-(d-1)$, and, by a direct calculation, $g_{F}(0,1)=g_{F}(1,0)=0$, thus:

$$
-1 / 2=B(F)=C_{d, 1} .
$$

\section{References}

[1] Bedford, E., Taylor, B. A., The Dirichlet problem for a complex Monge-Ampère equation. Bull. Amer. Math. Soc. 82 (1976), no. 1, 102-104.

[2] Berteloot, F., Mayer, V.: Rudiments de dynamique holomorphe. Cours Spécialisés (Paris) 7. Paris. Société Matématique de France. 160 p. (2001)

[3] Briend, J.-Y., Duval, J.: Exposants de Liapounoff et distribution des points périodiques d'un endomorphisme de $\mathbf{C P}^{k}$. Acta Math. 182 (1999), no. 2, 143-157.

[4] Brolin, H.: Invariant sets under iteration of rational functions. Ark. Mat. 6, 103-144 (1965). 
[5] Chen, X.: The space of Kähler metrics. J. Differ. Geom. 56, No.2, 189-234 (2000)

[6] Demailly, J.P.: Mesures de Monge-Ampére et caractérisation géométrique des variétés algébriques affines. Mém. Soc. Math. France (N.S.) No. 19 (1985), 124 pp.

[7] Demailly, J.P.:Regularization of closed positive currents and intersection theory, J. Algebraic Geometry 1, 361-406 (1992)

[8] DeMarco, L.: Dynamics of rational maps: Lyapunov exponents, bifurcations, and capacity. Math. Ann. 326, No.1, 43-73 (2003)

[9] DeMarco, L.: Dynamics of rational maps: A current on the bifurcation locus. Math. Res. Lett. 8, No.1-2, 57-66 (2001)

[10] Dinh, T.C., Sibony, N.: Dynamique des applications d'allure polynomiale. J. Math. Pures Appl., IX. Sér. 82, No.4, 367-423 (2003)

[11] Dold, A., Lectures on algebraic topology, Springer, Berlin (1972).

[12] Fornaess, J.E., Sibony, N.: Dynamics of $\mathbf{P}^{2}$ (examples). Lyubich, M. (ed.) et al., Laminations and foliations in dynamics, geometry and topology. Proceedings of the conference held at SUNY at Stony Brook, USA, May 18-24, 1998. Providence, AMS. Contemp. Math. 269, 47-85 (2001)

[13] Gelfand I. M., Kapranov M. M., Zelevinsky A.V., Discriminants, resultants, and multidimensional determinants. Birkhäuser Boston, Inc., Boston, MA, 1994. x+523

[14] Jonsson, M.: Sums of Lyapunov exponents for some polynomial maps of $\mathbf{C}^{2}$. Ergodic Theory Dyn. Syst. 18, No.3, 613-630 (1998)

[15] Mane, R.: The Hausdorff dimension of invariant probabilities of rational maps. Dynamical systems, Proc. Symp., Valparaiso/Chile 1986, Lect. Notes Math. 1331, 86-117 (1988)

[16] Mane, R., Sad, P., Sullivan, D.: On the dynamics of rational maps. Ann. Sci. Ec. Norm. Supér., IV. Sér. 16, 193-217 (1983)

[17] Manning, A.: The dimension of the maximal measure for a polynomial map. Ann. Math. (2) 119, 425-430 (1984)

[18] McMullen, C.T.: Complex dynamics and renormalization. Annals of Mathematics Studies. 135. Princeton, NJ: Univ. Press,. vii, 214 p. (1995).

[19] Milnor J.W., Geometry and dynamics of quadratic rational maps. With an appendix by the author and Lei Tan. Experiment. Math. 2 (1993), no. 1, 37-83

[20] Milnor, J.: Dynamics in one complex variable. Introductory lectures. 2nd ed. Wiesbaden: Vieweg. vii, 257 p. (2000)

[21] Milnor J.W., On Lattès maps. Stony Brook IMS Preprint n.2004/01, Math. ArXiv DS/0402147 
[22] Przytycki, F.: Hausdorff dimension of harmonic measure on the boundary of an attractive basin for a holomorphic map. Invent. Math. 80, 161-179 (1985)

[23] Ruelle, D., An inequality for the entropy of differentiable maps. Bol. Soc. Brasil. Mat. 9 (1978), no. 1, 83-87

[24] Semmes, S.: Complex Monge-Ampère equations and symplectic manifolds. Amer. J. Math., 114 (1992), 495-550

[25] Shishikura, M.: On the quasiconformal surgery of rational functions. Ann. Sci. Ec. Norm. Supér., IV. Sér. 20, No.1, 1-29 (1987)

[26] Sibony, N.: Dynamique des applications rationnelles de $\mathbf{P}^{k}$. Cerveau, Dominique et al., Dynamique et géométrie complexes. Paris: Société Mathématique de France. Panor. Synth. 8, 97-185 (1999)

[27] Silverman, J.H., The space of rational maps on $\mathbf{P}^{1}$. Duke Math. J. 94 (1998), 1, $41-77$.

[28] Siu, Y.T.: Analyticity of sets associated to Lelong numbers and the extension of closed positive currents. Invent. Math. 27, 53-156 (1974).

[29] Tortrat, P.: Aspects potentialistes de l'itération des polynômes. Théorie du potentiel, Sémin. Paris, No.8, Lect. Notes Math. 1235, 195-209 (1987)

Giovanni Bassanelli, Dipartimento di Matematica, Università di Parma, Parco Area delle Scienze, 153/A - I- 43100 Parma, Italia.

Email: giovanni.bassanelli@unipr.it

François Berteloot, Université Paul Sabatier MIG. Laboratoire Emile Picard UMR 5580. F-31062 Toulouse Cedex 9 France.

Email: berteloo@picard.ups-tlse.fr 